\title{
On a Model of Magnetization Switching by Spin-Polarized Current
}

\author{
K. Hamdache* ${ }^{*}$ D. Hamroun ${ }^{\dagger}$ and M. TiliouA ${ }^{\ddagger}$ \\ * Centre de Mathématiques Appliquées, \\ CNRS UMR 7641, Ecole Polytechnique, \\ 91128 Palaiseau Cedex, France \\ E-mail: hamdache@cmapx.polytechnique.fr \\ †Département de Mathématiques, Université USTHB, \\ BP 32 Al Alia, Bab Ezzouar, Alger, Algérie \\ E-mail: djamroun@yahoo.fr \\ $\ddagger$ Laboratoire de Modélisation et Calcul (LMC-IMAG), \\ CNRS UMR 5523, Université Joseph Fourier, \\ BP 53, 38041 Grenoble Cedex 9, France \\ E-mail: Mouhcine.Tilioua@imag.fr
}

Received November 12, 2004

Revised October 12, 2005

\begin{abstract}
This paper is concerned with global existence of weak solutions to a model equations of magnetization reversal by spin-polarized current in a layer introduced in [19]. The local magnetization of the ferromagnet satisfies the usual Landau-Lifshitz equation which is coupled to the nonlinear heat equation satisfied by the spin accumulation field defined in all the layer. The coupling is due to the contact interaction energy. We use an hyperbolic regularization method with penalization of the saturation constraint satisfied by the local magnetization to prove global existence result, in any finite time interval, of weak solutions with finite energy. We present other models of equations describing the magnetization switching by spin-polarized current and show that our method can be used to solve them.

Key words: ferromagnets, spin-polarized current, spin accumulation, magnetization switching
\end{abstract}

\section{Introduction}

The recent discovery that a spin-polarized electrical current can apply a large torque to a ferromagnet through direct transfer of spin angular momentum, offers the possibility of manipulating magnetic-device elements without applying any magnetic fields. Magnetic multilayers, devices which consist of alternating nanometerscale-thick films of magnetic and nonmagnetic materials, have been the focus of scientific and technological interest since the discovery of their giant magnetoresistance. While the effect of the magnetic layers on the current has been widely studied, the controverse phenomenon, the effect of the current on the orientation of the magnetic layers, has received less attention. Recent theories $[12,2]$ predict that a large spin-polarized current passing perpendicularly through the layers can exert torques on the magnetic moments in the layers, due to direct transfer of spin angular momentum from the conduction of electrons to the magnets. Convincing experiments have been now achieved and several theoretical approaches, extending the initial theory, have been recently developed for the interpretation of the existing 
experimental data. However the current density needed in the existing experiments is still relatively high, of the order of $10^{7}-10^{9} \mathrm{~A} / \mathrm{cm}^{2}$, and a better understanding of the spin transfer mechanisms seems necessary to obtain a significant reduction of the current density. Another effect of the same type but probably requiring a smaller current density is the displacement of a domain wall by spin transfer from a spin-polarized current. It was found experimentally that the current-induced spin torque has many unique properties. Among them, the spin torque distorts domain structures, drives domain walls out of equilibrium, makes magnetization switching from one direction to another, and creates spin excitations. While several theoretical models have been put forward to formulate the spin torque, the exact form is still the subject of much debate. These theories suggest an interesting magnetization relaxation process caused by a spin-polarized current. A conventional physical picture consists of a magnetic junction with two ferromagnetic layers one of which (layer 1 ) is pinned and the other (layer 2) is free. Very thin spacer layer exists between the layers 1 and 2 . The layer 2 has finite thickness and contacts at the second end with some nonmagnetic conductor (layer 3). The spin-polarized current may inject (or extract) nonequilibrium spins into layer 2. At a sufficiently large current density, the effective field leads to the magnetization reversal of the layer 2 . In order to focus further on these phenomena which have the important consequence that they can act as an additional magnetic field on the magnetic background, one assumes that when the bilinear or biquadratic interlayer exchange coupling exists, their effects are negligibles or one chooses the spacer layer thick enough to reduce the magnetic coupling between ferromagnets. We refer respectively to [7] and [6] for some mathematical results on these effects.

The main model equation we consider in this work is introduced by Zhang, Levy and Fert [19]. The effective magnetic field $\mathcal{H}_{e}$ is given by $\mathcal{H}_{e}=\mathcal{H}_{L L G}+J \boldsymbol{m}$ where $J \boldsymbol{m}$ corresponds to the contact interaction energy which is of the type $-J \int_{\Omega} \boldsymbol{m}$. $\boldsymbol{M} d x$ and $\mathcal{H}_{L L G}$ is the classical effective magnetic field in the micromagnetic theory. The spin accumulation field $\boldsymbol{m}$ satisfies to a nonlinear heat equation coupled to the LLG equation. The main difference for the new magnetization equation relies in the fact that the energy of the system is not conserved. So, we consider the coupled system for $\boldsymbol{m}$ and the local magnetization $\boldsymbol{M}$ on the finite time interval $[0, T]$ for any $T>0$ fixed.

Other models appear in the recent publications in the field of physics to describe the magnetization reversal by current injection after the pioneer contribution of Slonczewski [12] and Berger [2]. Some of them are [13], [1], [17] and [18] for a different approach. There is also other models, close to the previous ones, to describe the motion of domain wall by spin polarized current see [17]. In the last section of this work we recall these models equations and discuss their mathematical approaches.

Having briefly discussed the general context of this paper, let us go into its structure. In Section 2, we set down the model equations and the initial-boundary conditions for spin accumulation and magnetization. Section 3 is dedicated to our main results. We investigate the global existence of weak solutions to the coupled 
problem for spin accumulation and local magnetization dynamics. We first give formal estimates and then by regularization procedures we prove global existence of weak solutions in the time interval $[0, T]$ for all fixed and finite $T$. In Section 4, we discuss the case of stationary spin accumulation equation. We prove global existence of weak solutions by proceeding along the lines of the proof given in Section 3. The last section is devoted to some other models appearing in the recent papers. We show that our method can be performed to establish global existence theory for weak solutions.

In the sequel we use the following notations. If $\mathrm{D}$ is an open and regular set of $\mathbb{R}^{n}, \mathbb{L}^{2}(\mathrm{D})$ is the vectorial Lebesgue space $\left(L^{2}(\mathrm{D})\right)^{3}$ with norm and scalar product denoted respectively by $|\cdot|_{\mathrm{D}}$ and $(\cdot ; \cdot)_{\mathrm{D}}$. The Hilbert space $\mathbb{H}^{1}(\mathrm{D})$ is the usual Sobolev space $\left(H^{1}(\mathrm{D})\right)^{3}$. If $\mathrm{D}$ is a bounded domain of $\mathbb{R}^{n},|\mathrm{D}|$ will denote its Lebesgue measure.

\section{The Model Equations}

The calculations presented in this paper combine phenomenological constitutive equations for the electric current and the spin current with a Landau-LifshitzGilbert equation generalized to include spin-transfer torque. We adopt the model equations of magnetization switching by spin current proposed by [19], [11]. We also refer to [9] for the same model equations. Let $\left(\boldsymbol{e}_{1}, \boldsymbol{e}_{2}, \boldsymbol{e}_{3}\right)$ be the canonical basis of $\mathbb{R}^{3}$ and let $\widehat{\Omega}$ be an open, bounded and regular domain of $\mathbb{R}^{2}$ with generic point $\widehat{x}=(y, z)$. We denote by $x$ the abscissa of a point $X=(x, \widehat{x})$ of $\mathbb{R}^{3}, \Omega^{0}=(-h, h) \times \widehat{\Omega}$ denotes the nonmagnetic spacer $(N), \Omega^{1}=(h, l) \times \widehat{\Omega}$ and $\Omega^{2}=(-L,-h) \times \widehat{\Omega}$ the ferromagnetic materials $(F)$. We consider a magnetic junction of the type $F / N / F$ represented by the horizontal cylinder $\mathcal{O}=\Omega^{1} \cup \Omega^{0} \cup \Omega^{2}$. At the plane $x=-L$ an electric current $j_{e}$ is injected in the direction $\boldsymbol{e}_{1}$ and flows in the domain $I=[-L, l]$. Then the background magnetization receives a spin torque. This torque leads to spin accumulation in $N$ and in the regions of $F$ close to the $F / N$ and $N / F$ interfaces. If one denotes by $\boldsymbol{m}(t, x)$ the spin accumulation vector in the domain $\mathcal{O}$ for $t \geq 0$ and $x \in I$, then the magnetization current $\boldsymbol{j}_{m}$, in the case where the local magnetization $M$ is assumed to be uniform in $\Omega=\Omega^{1} \cup \Omega^{2}$ (with respect to the variable $X$ ), is given by the expression

$$
\boldsymbol{j}_{m}=\beta j_{e} \boldsymbol{M}-2 D_{0}\left(\partial_{x} \boldsymbol{m}-\beta \beta^{\prime} \boldsymbol{M}\left(\boldsymbol{M} \cdot \partial_{x} \boldsymbol{m}\right)\right) .
$$

Here, $\beta>0$ and $\beta^{\prime}>0$ represent the spin polarization parameters, $D_{0}>0$ the diffusion parameter. Theoretical analysis has been mostly confined to the case where the magnetization $\boldsymbol{M}$ of the layers is uniform. If $\boldsymbol{M}$ is not uniform in $\Omega$ then we can extend the expression (1) by setting

$$
\boldsymbol{j}_{m}=\beta j_{e}\langle\boldsymbol{M}\rangle-2 D_{0}\left(\partial_{x} \boldsymbol{m}-\beta \beta^{\prime}\langle\boldsymbol{M}\rangle\left(\langle\boldsymbol{M}\rangle \cdot \partial_{x} \boldsymbol{m}\right)\right)
$$


where $\langle\boldsymbol{M}\rangle$ is the mean value of $\boldsymbol{M}$ in $\widehat{\Omega},\langle\boldsymbol{M}\rangle=|\widehat{\Omega}|^{-1} \int_{\widehat{\Omega}} \boldsymbol{M}(t, x, \widehat{x}) \mathrm{d} \widehat{x}$. Hence, the spin accumulation is governed for $t \geq 0$ and $x \in I$, by the equation see [19], [11]

$$
\frac{1}{d^{2}} \partial_{t} \boldsymbol{m}-\partial_{x}\left(\mathcal{A}(\langle\boldsymbol{M}\rangle)\left(\partial_{x} \boldsymbol{m}\right)\right)+\frac{1}{\lambda_{J}^{2}} \boldsymbol{m} \times\langle\boldsymbol{M}\rangle+\frac{1}{\lambda_{\mathrm{sf}}^{2}} \boldsymbol{m}=-\frac{\beta}{d^{2}} \partial_{x}\left(j_{e}\langle\boldsymbol{M}\rangle\right)
$$

where we defined the matrix $\mathcal{A}(\langle\boldsymbol{M}\rangle)$ by

$$
\mathcal{A}(\langle\boldsymbol{M}\rangle)(\xi)=\xi-\beta \beta^{\prime}(\langle\boldsymbol{M}\rangle \cdot \xi)\langle\boldsymbol{M}\rangle
$$

for all $\xi \in \mathbb{R}^{3}$. The constants appearing in the equation are given by

$$
d=\sqrt{2 D_{0}}, \quad \lambda_{\mathrm{sf}}=d \sqrt{\tau_{\mathrm{sf}}}, \quad \lambda_{J}=d \sqrt{\hbar / J},
$$

where $\tau_{\mathrm{sf}}$ is the spin-flip relaxation time of the conduction electron, $J>0$ is the exchange interaction constant and $\hbar$ is the Planck constant.

The equation (3) is completed by initial and boundary conditions. First, we assume that the spin accumulation $\boldsymbol{m}$ as well as the magnetization current $\boldsymbol{j}_{m}$ are continuous across the interfaces $x=-h$ and $x=h$ (notice that $\boldsymbol{M}=0$ in $\Omega^{0}$ ). We have the transmission boundary conditions

$$
[\boldsymbol{m}(t, \cdot)]_{-h}=[\boldsymbol{m}(t, \cdot)]_{h}=0, \quad\left[\boldsymbol{j}_{m}(t, \cdot)\right]_{-h}=\left[\boldsymbol{j}_{m}(t, \cdot)\right]_{h}=0
$$

where we use the notation $[u(t, \cdot)]_{x_{0}}=u\left(t, x_{0}+0\right)-u\left(t, x_{0}-0\right)$ for a function $u$. The electric current $j_{e}(t, x)$ is a given function defined for $t \geq 0$ in the interval $-L \leq x \leq l$ and is linked to the spin accumulation $\boldsymbol{m}$ by (2). The boundary conditions for $\boldsymbol{m}$ at the interfaces $x=-L$ and $x=l$ take the form

$$
\left(\mathcal{A}(\langle\boldsymbol{M}\rangle)\left(\partial_{x} \boldsymbol{m}\right)(t, \cdot)-\frac{\beta}{d^{2}} j_{e}\langle\boldsymbol{M}\rangle(t, \cdot)\right)_{\mid x=-L, l}=0 .
$$

The spin accumulation equation is coupled to the local magnetization $\boldsymbol{M}(t, x, \widehat{x})$ which satisfies the LLG equation in $\mathbb{R}^{+} \times \Omega$ see [8], [3] for example

$$
\partial_{t} \boldsymbol{M}-\alpha \boldsymbol{M} \times \partial_{t} \boldsymbol{M}=-\gamma \boldsymbol{M} \times\left(\mathcal{H}_{e}+J \boldsymbol{m}\right)
$$

where $\boldsymbol{J} \boldsymbol{m}$ is the field associated with the contact interaction between the local moment and the spin accumulation. The parameters $\gamma>0$ and $\alpha>0$ are respectively the gyromagnetic and the Gilbert damping parameters. The effective magnetic field $\mathcal{H}_{e}$ contains the contribution of the magnetostatic, the volume anisotropy and the magnetic field associated with the exchange energy

$$
\mathcal{H}_{e}(\boldsymbol{M})=\nabla \cdot(a \nabla \boldsymbol{M})+\nabla \varphi-\nabla_{\boldsymbol{M}} \psi(\boldsymbol{M})
$$

where the exchange function $a(X)$ takes two positive values $a_{1}>0$ in $\Omega^{1}$ and $a_{2}>0$ in $\Omega^{2}$ and where $\varphi(t, X)$ satisfies in $\mathbb{R}^{+} \times \mathbb{R}^{3}$ the stray field equation

$$
\nabla \cdot(\nabla \varphi+\chi(\Omega) M)=0
$$


and $\nabla_{M} \psi$ is the volume anisotropy field associated with a regular function $\psi \in$ $C^{2}\left(\mathbb{R}^{3}\right)$ satisfying $\psi(X) \geq 0$ and $\left|D^{2} \psi(X)\right| \leq C$ for all $X \in \mathbb{R}^{3}$.

To end this description of the model equations, let us rewrite the initialboundary conditions satisfied by the field $(\boldsymbol{m}, \boldsymbol{M})$. We have for $\boldsymbol{m}$

$$
\boldsymbol{m}(0, x)=\boldsymbol{m}_{0}(x) \text { in } I, \quad j_{\boldsymbol{m}}(t,-L)=j_{\boldsymbol{m}}(t, l)=0 \text { in } \mathbb{R}^{+}
$$

and for $M$

$$
\left\{\begin{array}{l}
\boldsymbol{M}(0, X)=\boldsymbol{M}_{0}(X),\left|\boldsymbol{M}_{0}(X)\right|^{2}=1 \text { a.e. in } \Omega \\
\boldsymbol{M}(t, h+0, \widehat{x})=\boldsymbol{M}(t,-h-0, \widehat{x}) \text { in } \mathbb{R}^{+} \times \widehat{\Omega} \\
\left(\boldsymbol{M} \times a_{1} \partial_{x} \boldsymbol{M}\right)(t, h+0, \widehat{x})=\left(\boldsymbol{M} \times a_{2} \partial_{x} \boldsymbol{M}\right)(t,-h-0, \widehat{x}) \text { in } \mathbb{R}^{+} \times \widehat{\Omega} \\
\boldsymbol{M} \times(a \nabla \cdot n) \boldsymbol{M}=0 \text { on } \mathbb{R}^{+} \times\left(\partial \Omega \backslash\left(\Gamma^{1} \cup \Gamma^{2}\right)\right)
\end{array}\right.
$$

where we set $\Gamma^{1}=\{(h, \widehat{x}), \widehat{x} \in \widehat{\Omega}\}, \Gamma^{2}=\{(-h, \widehat{x}), \widehat{x} \in \widehat{\Omega}\}$ and $n$ is the unit outward normal to the boundary $\partial \Omega$ of $\Omega$. Finally, the magnetization $\boldsymbol{M}(t, X)$ satisfies the saturation condition

$$
|\boldsymbol{M}(t, X)|^{2}=1 \text { a.e. in } \mathbb{R}^{+} \times \Omega .
$$

As it is said in the introduction, we may use more complex boundary conditions coupling the interfaces $z= \pm h$ as the bilinear exchange one see [7] or the biquadratic exchange one see [6] for example.

In the next section we discuss the model equations (3)-(11)-(8)-(12).

\section{Global Weak Solutions to the Problem}

Our starting point is to give formally the a priori estimates satisfied by the solutions of the system (3)-(11)-(8)-(12).

\subsection{Formal estimates}

Any regular solution to the LLG equations (8) satisfies the relation

$$
\alpha\left|\partial_{t} \boldsymbol{M}\right|^{2}=\gamma\left(\mathcal{H}_{e}+J \boldsymbol{m}\right) \cdot \partial_{t} \boldsymbol{M}
$$

which leads after integration over $\Omega$ to

$$
\frac{1}{2} \frac{\mathrm{d}}{\mathrm{d} t} \mathcal{E}(\boldsymbol{M})+\frac{\alpha}{\gamma}\left|\partial_{t} \boldsymbol{M}\right|_{\Omega}^{2}=J \int_{\Omega} \boldsymbol{m} \cdot \partial_{t} \boldsymbol{M} \mathrm{d} X
$$

where $\mathcal{E}(\boldsymbol{M})$ is the energy of the system given by

$$
\mathcal{E}(\boldsymbol{M})=\int_{\Omega} a|\nabla \boldsymbol{M}|^{2} \mathrm{~d} X+\int_{\mathbb{R}^{3}}|\nabla \varphi|^{2} \mathrm{~d} X+2 \int_{\Omega} \psi(\boldsymbol{M}) \mathrm{d} X .
$$

By Cauchy-Schwarz inequality we get the estimate

$$
\frac{1}{2} \frac{\mathrm{d}}{\mathrm{d} t} \mathcal{E}(\boldsymbol{M})+\frac{\alpha}{2 \gamma}\left|\partial_{t} \boldsymbol{M}\right|_{\Omega}^{2} \leq \frac{\gamma J^{2}|\widehat{\Omega}|}{2 \alpha}|\boldsymbol{m}|_{I}^{2}
$$


Integration with respect to the time variable $t$ leads to

$$
\mathcal{E}(\boldsymbol{M}(t))+\frac{\alpha}{\gamma} \int_{0}^{t}\left|\partial_{t} \boldsymbol{M}(s)\right|_{\Omega}^{2} \mathrm{~d} s \leq \mathcal{E}\left(\boldsymbol{M}_{0}\right)+\frac{\gamma J^{2}|\widehat{\Omega}|}{\alpha} \int_{0}^{t}|\boldsymbol{m}(s)|_{I}^{2} \mathrm{~d} s
$$

where $\mathcal{E}\left(\boldsymbol{M}_{0}\right)$ is the initial energy defined by

$$
\mathcal{E}\left(\boldsymbol{M}_{0}\right)=\int_{\Omega} a\left|\nabla \boldsymbol{M}_{0}\right|^{2} \mathrm{~d} X+\int_{\mathbb{R}^{3}}\left|\nabla \varphi_{0}\right|^{2} \mathrm{~d} X+2 \int_{\Omega} \psi\left(\boldsymbol{M}_{0}\right) \mathrm{d} X
$$

where $\varphi_{0}$ satisfies the stray field equation associated with $\boldsymbol{M}_{0}$.

Let us consider for $\boldsymbol{M}$ fixed the heat equation satisfied by $\boldsymbol{m}$. We have the energy estimate

$$
\frac{1}{2 d^{2}} \frac{\mathrm{d}}{\mathrm{d} t}|\boldsymbol{m}|_{I}^{2}+\frac{1}{\lambda_{\mathrm{sf}}^{2}}|\boldsymbol{m}|_{I}^{2}+\left(\mathcal{A}(\langle\boldsymbol{M}\rangle) \partial_{x} \boldsymbol{m} ; \partial_{x} \boldsymbol{m}\right)_{\mathbb{L}^{2}(I)}=\frac{\beta}{d^{2}} \int_{I} j_{e}\langle\boldsymbol{M}\rangle \cdot \partial_{x} \boldsymbol{m} \mathrm{d} x
$$

Assuming the parameters $\beta$ and $\beta^{\prime}$ are such that

$$
1-\beta \beta^{\prime}>0
$$

then the matrix $\mathcal{A}(\langle\boldsymbol{M}\rangle)$ satisfies

$$
\left\{\begin{array}{l}
\mathcal{A}(\langle\boldsymbol{M}\rangle)(\xi) \cdot \xi \geq\left(1-\beta \beta^{\prime}\right)|\xi|^{2} \\
|\mathcal{A}(\langle\boldsymbol{M}\rangle)(\xi)| \leq\left(1+\beta \beta^{\prime}\right)|\xi|
\end{array}\right.
$$

for all $\xi \in \mathbb{R}^{3}$. We deduce, by using the saturation condition $|\boldsymbol{M}(t, X)|^{2}=1$, the inequality

$$
\begin{aligned}
& \frac{1}{2 d^{2}}|\boldsymbol{m}(t)|_{I}^{2}+\frac{1}{\lambda_{\mathrm{sf}}^{2}} \int_{0}^{t}|\boldsymbol{m}(s)|_{I}^{2} \mathrm{~d} s+\frac{1-\beta \beta^{\prime}}{2} \int_{0}^{t}\left|\partial_{x} \boldsymbol{m}\right|_{I}^{2} \mathrm{~d} s \\
& \leq \frac{1}{2 d^{2}}\left|\boldsymbol{m}_{0}\right|_{I}^{2}+\frac{\beta^{2}}{2 d^{4}\left(1-\beta \beta^{\prime}\right)} \int_{0}^{t}\left|j_{e}\right|_{I}^{2} \mathrm{~d} s .
\end{aligned}
$$

Estimates (16) and (21) lead to a bound for the energy of the local magnetization $\boldsymbol{M}$ on the interval $[0, T]$ for $T$ fixed and finite.

REMARK 3.1. Notice that the contact interaction energy satisfies the inequality

$$
\left|\int_{\Omega} \boldsymbol{m} \cdot \boldsymbol{M} d X\right| \leq|\widehat{\Omega}|^{1 / 2}\left(\int_{I}|\boldsymbol{m}|^{2} d x\right)^{1 / 2} .
$$

It follows that energy is controlled by the contribution of the spin accumulation energy.

We shall prove a global existence result for weak solutions to problem (3)(11)-(8)-(12). We set $Q=(0, T) \times \Omega$ and $\omega=(0, T) \times I$ for $T>0$ fixed. 


\subsection{Regularization: the intermediary problem}

We proceed along the lines of the method introduced in [5]. See also [6]. The idea is to write, by using the saturation condition, the LLG equation in the form $\boldsymbol{M} \times\left((1 / \gamma) \boldsymbol{M} \times \partial_{t} \boldsymbol{M}+(\alpha / \gamma) \partial_{t} \boldsymbol{M}-\mathcal{H}_{e}-\boldsymbol{J} \boldsymbol{m}-(q(|\boldsymbol{M}|) \boldsymbol{M})\right)=0$ for any function $q$ of $|\boldsymbol{M}|$. Hence, for a small fixed parameter $\varepsilon>0$, we introduce the vector function $U^{\varepsilon}$ satisfying the intermediary problem

$$
\left\{\begin{array}{l}
\frac{\alpha}{\gamma} \partial_{t} U^{\varepsilon}-\nabla \cdot\left(a \nabla U^{\varepsilon}\right)+\frac{1}{\varepsilon^{2}} \nabla_{U}\left(p\left(\left|U^{\varepsilon}\right|\right)\right)=\mathcal{L}\left(U^{\varepsilon}\right)+\mathcal{R}^{\varepsilon}\left(U^{\varepsilon}, \partial_{t} U^{\varepsilon}\right)+J \boldsymbol{m}^{\varepsilon} \text { in } Q \\
U^{\varepsilon}(0, X)=\boldsymbol{M}_{0}(X) \text { in } \Omega,\left|\boldsymbol{M}_{0}(X)\right|^{2}=1 \text { a.e. in } \Omega \\
U^{\varepsilon}(t, h+0, \widehat{x})=U^{\varepsilon}(t,-h-0, \widehat{x}) \text { in }(0, T) \times \widehat{\Omega} \\
a_{1} \partial_{x} U^{\varepsilon}(t, h+0, \widehat{x})=a_{2} \partial_{x} U^{\varepsilon}(t,-h-0, \widehat{x}) \text { in }(0, T) \times \widehat{\Omega} \\
(a \nabla \cdot n) U^{\varepsilon}=0 \text { on }(0, T) \times\left(\partial \Omega \backslash\left(\Gamma^{1} \cup \Gamma^{2}\right)\right) .
\end{array}\right.
$$

The spin accumulation field $\boldsymbol{m}^{\varepsilon}$ satisfies the heat equation

$$
\left\{\begin{array}{l}
\frac{1}{d^{2}} \partial_{t} \boldsymbol{m}^{\varepsilon}-\partial_{x}\left(\mathcal{A}^{\varepsilon}\left(\left\langle U^{\varepsilon}\right\rangle\right)\left(\partial_{x} \boldsymbol{m}^{\varepsilon}\right)\right)+\frac{1}{\lambda_{\mathrm{sf}}^{2}} \boldsymbol{m}^{\varepsilon} \\
=-\frac{1}{\lambda_{J}^{2}} \boldsymbol{m}^{\varepsilon} \times\left\langle U^{\varepsilon}\right\rangle-\frac{\beta}{d^{2}} \partial_{x}\left(j_{e}\left\langle U^{\varepsilon}\right\rangle\right) \text { in } \omega \\
\boldsymbol{m}^{\varepsilon}(0, x)=\boldsymbol{m}_{0}(x) \text { in } I \\
j^{\varepsilon}\left(U^{\varepsilon}, \boldsymbol{m}^{\varepsilon}\right)(t,-L)=j^{\varepsilon}\left(U^{\varepsilon}, \boldsymbol{m}^{\varepsilon}\right)(t, l)=0 \text { in }(0, T)
\end{array}\right.
$$

where we set

$$
\begin{aligned}
& \mathcal{L}\left(U^{\varepsilon}\right)=\nabla \varphi^{\varepsilon}-\nabla_{U} \psi\left(U^{\varepsilon}\right), \mathcal{R}^{\varepsilon}(V, W)=\frac{1}{\gamma} \mathcal{S}^{\varepsilon}(V) \times W \\
& \mathcal{S}^{\varepsilon}(\xi)=\xi /(\varepsilon+|\xi|), \quad \forall \xi \in \mathbb{R}^{3}, \mathcal{T}^{\varepsilon}(V)=\langle V\rangle /(\varepsilon+\langle|V|\rangle) \\
& p(r)=\left|\sqrt{1+r^{2}}-\sqrt{2}\right|^{2}, \quad r \in \mathbb{R}^{+} \\
& \mathcal{A}^{\varepsilon}(\langle V\rangle) \xi=\xi-\beta \beta^{\prime}\left(\mathcal{T}^{\varepsilon}(V) \cdot \xi\right) \mathcal{T}^{\varepsilon}(V), \forall \xi \in \mathbb{R}^{3} \\
& j^{\varepsilon}(V, \boldsymbol{m})=\beta j_{e}\langle V\rangle-d^{2} \mathcal{A}^{\varepsilon}(\langle V\rangle)\left(\partial_{x} \boldsymbol{m}\right) .
\end{aligned}
$$

Operator $\mathcal{L}$ is Lipschitz continuous from $\mathbb{L}^{2}(\Omega)$ into $\mathbb{L}^{2}(\Omega)$ by using the linearity of the stray field equation and the bound, $\left|D^{2} \psi(\xi)\right| \leq C$ for all $\xi$, of $\psi$. We have for all $U, V \in \mathbb{L}^{2}(\Omega)$ the estimate

$$
|\mathcal{L}(U)-\mathcal{L}(V)|_{\Omega} \leq C|U-V|_{\Omega}
$$

where $C>0$ represents various constants which are independent of $\varepsilon$. Moreover, operator $\mathcal{R}^{\varepsilon}\left(U^{\varepsilon}, \partial_{t} U^{\varepsilon}\right)$ satisfies locally the orthogonality property $\mathcal{R}^{\varepsilon}\left(U^{\varepsilon}, \partial_{t} U^{\varepsilon}\right)$. $U^{\varepsilon}=\mathcal{R}^{\varepsilon}\left(U^{\varepsilon}, \partial_{t} U^{\varepsilon}\right) \cdot \partial_{t} U^{\varepsilon}=0$. As we have for all $r \geq 0$

$$
r^{2}-1=p(r)+2 \sqrt{2}\left(\sqrt{r^{2}+1}-\sqrt{2}\right)
$$


we can deduce the $\mathbb{L}^{2}(\Omega)$ bound for $U$ knowing the $L^{1}(\Omega)$ bound of $p(|U|)$. The matrix operator $\mathcal{A}^{\varepsilon}$ satisfies for all $\xi \in \mathbb{R}^{3}$ the bounds

$$
\mathcal{A}^{\varepsilon}(\langle V\rangle)(\xi) \cdot \xi \geq\left(1-\beta \beta^{\prime}\right)|\xi|^{2}, \quad\left|\mathcal{A}^{\varepsilon}(\langle V\rangle)(\xi)\right| \leq\left(1+\beta \beta^{\prime}\right)|\xi|
$$

and $\mathcal{T}^{\varepsilon}$ satisfies for all functions $U_{1}, U_{2} \in \mathbb{L}^{\infty}(Q)$, the estimates

$$
\left\{\begin{array}{l}
\left|\mathcal{T}^{\varepsilon}\left(U_{1}\right)\right| \leq 1,\left|\mathcal{T}^{\varepsilon}\left(U_{1}\right)-\mathcal{T}^{\varepsilon}\left(U_{2}\right)\right|_{\infty} \leq C_{\varepsilon}\left|U_{1}-U_{2}\right|_{\infty} \\
\left|\mathcal{T}^{\varepsilon}\left(U_{1}\right) \otimes \mathcal{T}^{\varepsilon}\left(U_{1}\right)-\mathcal{T}^{\varepsilon}\left(U_{2}\right) \otimes \mathcal{T}^{\varepsilon}\left(U_{2}\right)\right|_{\infty} \leq C_{\varepsilon}\left|U_{1}-U_{2}\right|_{\infty}
\end{array}\right.
$$

where $C_{\varepsilon}>0$ is a constant depending only of $\varepsilon,|\cdot|_{\infty}$ denotes the norm of the two spaces $\mathbb{L}^{\infty}(Q)$ and $\mathbb{L}^{\infty}(\omega)$ while $V \otimes W$ is the matrix $(V \otimes W)_{i, j}=V_{i} W_{j}$, $1 \leq i, j \leq 3$ for all vectors $V, W \in \mathbb{R}^{3}$. Moreover, for all $\xi_{1}, \xi_{2}, \eta_{1}, \eta_{2} \in \mathbb{R}^{3}$, we may write $\left(\mathcal{A}^{\varepsilon}\left(\eta_{1}\right) \xi_{1}-\mathcal{A}^{\varepsilon}\left(\eta_{2}\right) \xi_{2}\right) \cdot\left(\xi_{1}-\xi_{2}\right)=\mathcal{A}^{\varepsilon}\left(\eta_{1}\right)\left(\xi_{1}-\xi_{2}\right) \cdot\left(\xi_{1}-\xi_{2}\right)-$ $\beta \beta^{\prime}\left(\mathcal{T}^{\varepsilon}\left(\eta_{1}\right) \otimes \mathcal{T}^{\varepsilon}\left(\eta_{1}\right)-\mathcal{T}^{\varepsilon}\left(\eta_{2}\right) \otimes \mathcal{T}^{\varepsilon}\left(\eta_{2}\right)\right) \xi_{2} \cdot\left(\xi_{1}-\xi_{2}\right)$ and then we get for all functions $U_{1}, U_{2} \in \mathbb{L}^{\infty}(Q), V_{1}, V_{2} \in \mathbb{L}^{2}(\omega)$

$$
\begin{aligned}
& \left(\mathcal{A}^{\varepsilon}\left(\left\langle U_{1}\right\rangle\right) V_{1}-\mathcal{A}^{\varepsilon}\left(\left\langle U_{2}\right\rangle\right) V_{2} ; V_{1}-V_{2}\right)_{\omega} \\
& \geq \frac{\left(1-\beta \beta^{\prime}\right)}{2}\left|V_{1}-V_{2}\right|_{\omega}^{2}-\frac{\left(\beta \beta^{\prime}\right)^{2}}{2\left(1-\beta \beta^{\prime}\right)} C_{\varepsilon}^{2}\left|U_{1}-U_{2}\right|_{\infty}^{2}\left|V_{2}\right|_{\omega}^{2} .
\end{aligned}
$$

Let us give another property satisfied by operator $\mathcal{R}^{\varepsilon}$. Since $\mathcal{S}^{\varepsilon}$ is Lipschitz continuous with Lipschitz constant $C_{\varepsilon}$ then for all $U_{1}, U_{2} \in \mathbb{L}^{\infty}(Q), V_{1}, V_{2} \in \mathbb{L}^{2}(Q)$ we have $\gamma\left(\mathcal{R}^{\varepsilon}\left(U_{1}, V_{1}\right)-\mathcal{R}^{\varepsilon}\left(U_{2}, V_{2}\right) ; V_{1}-V_{2}\right)_{Q}=\left(\left(\mathcal{S}^{\varepsilon}\left(U_{1}\right)-\mathcal{S}^{\varepsilon}\left(U_{2}\right)\right) \times V_{2} ; V_{1}-V_{2}\right)_{Q}$ and then we get

$$
\left|\left(\mathcal{R}^{\varepsilon}\left(U_{1}, V_{1}\right)-\mathcal{R}^{\varepsilon}\left(U_{2}, V_{2}\right) ; V_{1}-V_{2}\right)_{Q}\right| \leq \frac{C_{\varepsilon}}{\gamma}\left|U_{1}-U_{2}\right|_{\infty}\left|V_{2}\right|_{Q}\left|V_{1}-V_{2}\right|_{Q} .
$$

\subsection{Solving the intermediary problem $(23)-(24)$}

Let us consider for $\nu>0$ fixed the hyperbolic regularization of (23)

$$
\left\{\begin{array}{l}
\nu^{2} \partial_{t}^{2} U_{\nu}^{\varepsilon}-\nabla \cdot\left(a \nabla U_{\nu}^{\varepsilon}\right)+\mathcal{B}^{\varepsilon}\left(\rho^{\nu} \star \widetilde{U}_{\nu}^{\varepsilon}, \partial_{t} U_{\nu}^{\varepsilon}\right) \\
=-\frac{1}{\varepsilon^{2}} \nabla_{U}\left(p\left(\left|U_{\nu}^{\varepsilon}\right|\right)\right)+\mathcal{L}\left(U_{\nu}^{\varepsilon}\right)+J \boldsymbol{m}_{\nu}^{\varepsilon} \text { in } Q \\
U_{\nu}^{\varepsilon}(0, X)=\boldsymbol{M}_{0}(X) \text { in } \Omega,\left|\boldsymbol{M}_{0}(X)\right|^{2}=1 \text { a.e., } \nu^{2} \partial_{t} U_{\nu}^{\varepsilon}(0, X)=0 \text { in } \Omega
\end{array}\right.
$$

with $U_{\nu}^{\varepsilon}$ satisfying the boundary condition of problem (23). We set

$$
\mathcal{B}^{\varepsilon}(U, V)=\frac{\alpha}{\gamma} V-\mathcal{R}^{\varepsilon}(U, V)
$$

$\widetilde{U}$ denotes the extension of $U$ by 0 outside $Q, \star$ is the convolution product with respect to $(t, X)$ and $\rho^{\nu}$ is a regularizing sequence. Notice that operator $\mathcal{B}^{\varepsilon}$ satisfies, locally for all $U$ and $V$, the property

$$
\mathcal{B}^{\varepsilon}(U, V) \cdot V=\frac{\alpha}{\gamma}|V|^{2} .
$$


The spin accumulation field $\boldsymbol{m}_{\nu}^{\varepsilon}$ satisfies

$$
\left\{\begin{array}{l}
\frac{1}{d^{2}} \partial_{t} \boldsymbol{m}_{\nu}^{\varepsilon}-\partial_{x}\left(\mathcal{A}^{\varepsilon}\left(\left\langle\rho^{\nu} \star \widetilde{U}_{\nu}^{\varepsilon}\right\rangle\right)\left(\partial_{x} \boldsymbol{m}_{\nu}^{\varepsilon}\right)\right)+\frac{1}{\lambda_{\text {sf }}^{2}} \boldsymbol{m}_{\nu}^{\varepsilon} \\
\quad=-\frac{1}{\lambda_{J}^{2}} \boldsymbol{m}_{\nu}^{\varepsilon} \times \mathcal{T}^{\varepsilon}\left(\rho^{\nu} \star \widetilde{U}_{\nu}^{\varepsilon}\right)-\frac{\beta}{d^{2}} \partial_{x}\left(j_{e} \mathcal{T}^{\varepsilon}\left(\rho^{\nu} \star \widetilde{U}_{\nu}^{\varepsilon}\right)\right) \text { in } \omega \\
\boldsymbol{m}_{\nu}^{\varepsilon}(0, x)=\boldsymbol{m}_{0}(x) \text { in } I \\
j_{\nu}^{\varepsilon}\left(U_{\nu}^{\varepsilon}, \boldsymbol{m}_{\nu}^{\varepsilon}\right)(t,-L)=j_{\nu}^{\varepsilon}\left(U_{\nu}^{\varepsilon}, \boldsymbol{m}_{\nu}^{\varepsilon}\right)(t, l)=0 \text { in }(0, T)
\end{array}\right.
$$

where $j_{\nu}^{\varepsilon}(U, \boldsymbol{m})=\beta j_{e} \mathcal{T}^{\varepsilon}\left(\rho^{\nu} \star \widetilde{U}\right)-d^{2} \mathcal{A}^{\varepsilon}\left(\left\langle\rho^{\nu} \star \widetilde{U}\right\rangle\right)\left(\partial_{x} \boldsymbol{m}\right)$.

We fix $\varepsilon$ and $\nu$. In the sequel of this paragraph, we will show existence of solutions for the regularized problem (32)-(35) by applying a fixed point procedure. Let $V \in L^{2}\left(0, T ; \mathbb{L}^{2}(\Omega)\right)$ be fixed, we consider now the spin accumulation equation (35) associated with $V$

$$
\left\{\begin{array}{l}
\frac{1}{d^{2}} \partial_{t} \boldsymbol{m}-\partial_{x}\left(\mathcal{A}^{\varepsilon}\left(\left\langle\rho^{\nu} \star \widetilde{V}\right\rangle\right)\left(\partial_{x} \boldsymbol{m}\right)\right)+\frac{1}{\lambda_{\text {sf }}^{2}} \boldsymbol{m} \\
\quad=-\frac{1}{\lambda_{J}^{2}} \boldsymbol{m} \times \mathcal{T}^{\varepsilon}\left(\rho^{\nu} \star \widetilde{V}\right)-\frac{\beta}{d^{2}} \partial_{x}\left(j_{e} \mathcal{T}^{\varepsilon}\left(\rho^{\nu} \star \widetilde{V}\right)\right) \text { in } \omega \\
\boldsymbol{m}(0, x)=\boldsymbol{m}_{0}(x) \text { in } I \\
j_{\nu}^{\varepsilon}(V, \boldsymbol{m})(t,-L)=j_{\nu}^{\varepsilon}(V, \boldsymbol{m})(t, l)=0 \text { in }(0, T)
\end{array}\right.
$$

The solution of (36) is such that $\boldsymbol{m} \in L^{\infty}\left(0, T ; \mathbb{L}^{2}(I)\right), \partial_{x} \boldsymbol{m} \in L^{2}\left(0, T ; \mathbb{L}^{2}(I)\right)$ and $\partial_{t} \boldsymbol{m} \in L^{2}\left(0, T ; \mathbb{H}^{-1}(I)\right)$. Moreover, the following energy inequality holds

$$
\begin{aligned}
& \frac{1}{2 d^{2}}|\boldsymbol{m}(t)|_{I}^{2}+\frac{1}{\lambda_{\mathrm{sf}}^{2}} \int_{0}^{t}|\boldsymbol{m}(s)|_{I}^{2} \mathrm{~d} s+\frac{1-\beta \beta^{\prime}}{2} \int_{0}^{t}\left|\partial_{x} \boldsymbol{m}(s)\right|_{I}^{2} \mathrm{~d} s \\
& \leq \frac{1}{2 d^{2}}\left|\boldsymbol{m}_{0}\right|_{I}^{2}+\frac{\beta^{2}}{2 d^{4}\left(1-\beta \beta^{\prime}\right)} \int_{0}^{T}\left|j_{e}\right|_{I}^{2} \mathrm{~d} s .
\end{aligned}
$$

We define the map $\mathcal{Q}: V \in L^{2}\left(0, T ; \mathbb{L}^{2}(\Omega)\right) \mapsto \boldsymbol{m}=\mathcal{Q}(V)$ solution of (36). We have

Lemma 3.1. $\mathcal{Q}$ is Lipschitz continuous from $L^{2}\left(0, T ; \mathbb{L}^{2}(\Omega)\right)$ into $L^{2}\left(0, T ; \mathbb{L}^{2}(I)\right)$.

Proof. Let $V_{k} \in L^{2}\left(0, T ; \mathbb{L}^{2}(\Omega)\right)$ and $\boldsymbol{m}_{k}=\mathcal{Q}\left(V_{k}\right)$ be the solution of (36) associated with $V_{k}$ for $k=1,2$. We set $\boldsymbol{m}=\boldsymbol{m}_{1}-\boldsymbol{m}_{2}$ and $V=V_{1}-V_{2}$. It follows that $\boldsymbol{m}$ satisfies the equation

$$
\begin{aligned}
& \frac{1}{d^{2}} \partial_{t} \boldsymbol{m}-\partial_{x}\left(\mathcal{A}^{\varepsilon}\left(\left\langle\rho^{\nu} \star \widetilde{V}_{1}\right\rangle\right)\left(\partial_{x} \boldsymbol{m}_{1}\right)-\mathcal{A}^{\varepsilon}\left(\left\langle\rho^{\nu} \star \widetilde{V}_{2}\right\rangle\right)\left(\partial_{x} \boldsymbol{m}_{2}\right)\right)+\frac{1}{\lambda_{\mathrm{sf}}^{2}} \boldsymbol{m} \\
& =-\frac{1}{\lambda_{J}^{2}} \boldsymbol{m} \times \mathcal{T}^{\varepsilon}\left(\rho^{\nu} \star \widetilde{V}_{1}\right)-\frac{1}{\lambda_{J}^{2}} \boldsymbol{m}_{2} \times\left(\mathcal{T}^{\varepsilon}\left(\rho^{\nu} \star \widetilde{V}_{1}\right)-\mathcal{T}^{\varepsilon}\left(\rho^{\nu} \star \widetilde{V}_{2}\right)\right) \\
& \quad-\frac{\beta}{d^{2}} \partial_{x}\left(j_{e}\left(\mathcal{T}^{\varepsilon}\left(\rho^{\nu} \star \widetilde{V}_{1}\right)-\mathcal{T}^{\varepsilon}\left(\rho^{\nu} \star \widetilde{V}_{2}\right)\right)\right)
\end{aligned}
$$


with homogeneous initial and boundary conditions. Multiplying by $\boldsymbol{m}$, integrating by parts and using the property satisfied by $\mathcal{A}^{\varepsilon}$ we get

$$
\begin{aligned}
& \frac{1}{2 d^{2}}|\boldsymbol{m}(t)|_{I}^{2}+\frac{1}{2 \lambda_{\mathrm{sf}}^{2}} \int_{0}^{t}|\boldsymbol{m}(s)|_{I}^{2} \mathrm{~d} s+\frac{1-\beta \beta^{\prime}}{2} \int_{0}^{t}\left|\partial_{x} \boldsymbol{m}(s)\right|_{I}^{2} \mathrm{~d} s \\
& \leq C_{\varepsilon}^{2}\left|\rho^{\nu} \star \widetilde{V}\right|_{\infty}^{2} \int_{0}^{t}\left(\frac{1}{2} \lambda_{\mathrm{sf}}^{2} \lambda_{J}^{-2}\left|\boldsymbol{m}_{2}\right|_{I}^{2}+\frac{\beta^{2}\left|\beta^{\prime}\right|^{2}}{1-\beta \beta^{\prime}}\left|\partial_{x} \boldsymbol{m}_{2}\right|_{I}^{2}+\frac{\beta^{2}}{\left(1-\beta \beta^{\prime}\right) d^{4}}\left|j_{e}\right|_{I}^{2}\right) \mathrm{d} s .
\end{aligned}
$$

Finally arguing the fact that $\left|\rho^{\nu} \star \widetilde{V}\right|_{\infty} \leq C_{\nu}|V|_{Q}$ we deduce the continuity of the map $\mathcal{Q}$ from $L^{2}\left(0, T ; \mathbb{L}^{2}(\Omega)\right)$ into $L^{\infty}\left(0, T ; \mathbb{L}^{2}(I)\right) \cap L^{2}\left(0, T ; \mathbb{H}^{1}(I)\right)$. The Lipschitz continuity in these spaces is then a consequence of $(37)$.

Let us consider now for $V$ fixed in $L^{2}\left(0, T ; \mathbb{L}^{2}(\Omega)\right)$ the equation

$$
\left\{\begin{array}{l}
\nu^{2} \partial_{t}^{2} U-\nabla \cdot(a \nabla U)+\mathcal{B}^{\varepsilon}\left(\rho \star \widetilde{V}, \partial_{t} U\right)=-\frac{1}{\varepsilon^{2}} \nabla_{U}(p(|U|)) \\
\quad+\mathcal{L}(U)+J \mathcal{Q}(V) \text { in } Q \\
U(0, X)=M_{0}(X) \text { in } \Omega,\left|M_{0}(X)\right|^{2}=1 \text { a.e., } \nu^{2} \partial_{t} U(0, X)=0 \text { in } \Omega
\end{array}\right.
$$

with the same boundary conditions as in (23). Since the right-hand side of the equation is Lipschitz continuous (with respect to $U$ ) from $L^{2}\left(0, T ; \mathbb{L}^{2}(\Omega)\right.$ ) into $L^{2}\left(0, T ; \mathbb{L}^{2}(\Omega)\right)$ then the wave equation (40) admits a unique global weak solution $U$ with finite energy see [4], [16], [14], [10]. It satisfies the estimate

$$
\begin{aligned}
& \nu^{2}\left|\partial_{t} U(t)\right|_{\Omega}^{2}+\mathcal{E}(U(t))+\frac{2}{\varepsilon^{2}} \int_{\Omega} p(|U(t)|) \mathrm{d} X+\frac{\alpha}{\gamma} \int_{0}^{t}\left|\partial_{t} U(s)\right|_{\Omega}^{2} \mathrm{~d} s \\
& \leq \mathcal{E}\left(\boldsymbol{M}_{0}\right)+\frac{J^{2}|\widehat{\Omega}| \gamma}{\alpha} \int_{0}^{t}|\mathcal{Q}(V)(s)|_{I}^{2} \mathrm{~d} s .
\end{aligned}
$$

Let $\mathcal{K}$ be the map defined by $\mathcal{K}(V)=U, U$ solution of (40). We have the result

LEMmA 3.2. Operator $\mathcal{K}: L^{2}\left(0, T ; \mathbb{L}^{2}(\Omega)\right) \rightarrow L^{2}\left(0, T ; \mathbb{L}^{2}(\Omega)\right)$ is continuous and compact.

Proof. For $\nu$ and $\varepsilon$ fixed, operator $\mathcal{K}$ is bounded from $L^{2}\left(0, T ; \mathbb{L}^{2}(\Omega)\right)$ into the Banach space $\mathcal{X}=L^{\infty}\left(0, T ; \mathbb{H}^{1}(\Omega)\right) \cap H^{1}\left(0, T ; \mathbb{L}^{2}(\Omega)\right)$. By using Aubin's compactness lemma it follows that $\mathcal{X}$ is compactly embedded into $L^{2}\left(0, T ; \mathbb{L}^{2}(\Omega)\right)$ and then $\mathcal{K}$ is compact from $L^{2}\left(0, T ; \mathbb{L}^{2}(\Omega)\right)$ into itself. Let us prove that $\mathcal{K}$ is continuous. Let $V_{k} \in L^{2}\left(0, T ; \mathbb{L}^{2}(\Omega)\right) k=1,2$ and $U_{k}=\mathcal{K}\left(V_{k}\right)$ be the solution of (40). We set $V=V_{1}-V_{2}$ and $U=U_{1}-U_{2}$. Hence $U$ satisfies the equation

$$
\begin{aligned}
& \nu^{2} \partial_{t}^{2} U-\nabla \cdot(a \nabla U)+\mathcal{B}^{\varepsilon}\left(\rho^{\nu} \star \widetilde{V}_{1}, \partial_{t} U\right) \\
& =\mathcal{R}^{\varepsilon}\left(\rho^{\nu} \star \widetilde{V}_{1}, \partial_{t} U_{2}\right)-\mathcal{R}^{\varepsilon}\left(\rho^{\nu} \star \widetilde{V}_{2}, \partial_{t} U_{2}\right)-\frac{1}{\varepsilon^{2}}\left[\nabla_{U}\left(p\left(\left|U_{1}\right|\right)\right)\right. \\
& \left.\quad-\nabla_{U}\left(p\left(\left|U_{2}\right|\right)\right)\right]+\mathcal{L}\left(U_{1}\right)-\mathcal{L}\left(U_{2}\right)+J\left(\mathcal{Q}\left(V_{1}\right)-\mathcal{Q}\left(V_{2}\right)\right)
\end{aligned}
$$


with homogeneous initial condition and the same boundary conditions as in (23). Multiplying this equation by $\partial_{t} U$, integrating by parts, using the Lipschitz property of $\nabla_{U}(p(|U|))$ and $\nabla_{U} \psi$ and the property satisfied by $\mathcal{R}^{\varepsilon}$ we get

$$
\begin{aligned}
& \nu^{2}\left|\partial_{t} U(t)\right|_{\Omega}^{2}+|\sqrt{a} \nabla U(t)|_{\Omega}^{2}+|\nabla \varphi(t)|_{\Omega}^{2}+\frac{\alpha}{\gamma} \int_{0}^{t}\left|\partial_{t} U(s)\right|_{\Omega}^{2} \mathrm{~d} s \\
& \leq+\frac{3 J^{2}|\widehat{\Omega}| \gamma}{\alpha} \int_{0}^{t}\left|\mathcal{Q}\left(V_{1}\right)-\mathcal{Q}\left(V_{2}\right)\right|_{I}^{2} \mathrm{~d} s+\frac{3 C_{\varepsilon}^{2}}{\alpha}\left|\rho^{\nu} \star \widetilde{V}\right|_{\infty}^{2} \int_{0}^{T}\left|\partial_{t} U_{2}(s)\right|_{\Omega}^{2} \mathrm{~d} s \\
& \quad+\frac{3 C_{\varepsilon}^{2} \gamma}{\alpha} \int_{0}^{t}|U(s)|_{\Omega}^{2} \mathrm{~d} s
\end{aligned}
$$

where $\varphi$ is the solution of the stray equation associated with $U$. Next, using the inequality $|U(s)|^{2} \leq \int_{0}^{s}|U(\alpha)|^{2} \mathrm{~d} \alpha+\int_{0}^{t}\left|\partial_{t} U(\alpha)\right|^{2} \mathrm{~d} \alpha$ for all $0 \leq s \leq t \leq T$ we get the estimate $|U(s)|^{2} \leq\left(1+s e^{s}\right) \int_{0}^{t}\left|\partial_{t} U(\alpha)\right|^{2} \mathrm{~d} \alpha$ and finally the previous energy estimate implies the following

$$
\nu^{2}\left|\partial_{t} U(t)\right|_{\Omega}^{2} \leq C\left|\mathcal{Q}\left(V_{1}\right)-\mathcal{Q}\left(V_{2}\right)\right|_{\omega}^{2}+C_{\varepsilon, T}\left|\rho^{\nu} \star \widetilde{V}\right|_{\infty}^{2}+C_{\varepsilon, T} \int_{0}^{t}\left|\partial_{t} U(s)\right|_{\Omega}^{2} \mathrm{~d} s
$$

Then using Gronwall inequality we obtain

$$
\left|\partial_{t} U(t)\right|_{\Omega}^{2} \leq C_{\varepsilon, \nu, T}\left(\left|\mathcal{Q}\left(V_{1}\right)-\mathcal{Q}\left(V_{2}\right)\right|_{\omega}^{2}+\left|\rho^{\nu} \star \widetilde{V}\right|_{\infty}^{2}\right)
$$

where $C, C_{\varepsilon, T}$ and $C_{\varepsilon, \nu, T}$ are various positive constants. Finally since we have $\left|\rho^{\nu} \star \widetilde{V}\right|_{\infty} \leq C_{\nu}|V|_{L^{2}\left(0, T ; \mathbb{L}^{2}(\Omega)\right)}$ and operator $\mathcal{Q}$ is Lipschitz continuous by the previous lemma we conclude that $\left|\partial_{t} U(t)\right|_{\Omega}^{2} \leq C_{\varepsilon, \nu, T} \int_{0}^{T}|V(s)|_{\Omega}^{2}$ ds and $|U(t)|_{\Omega}^{2} \leq$ $C_{\varepsilon, \nu, T} \int_{0}^{T}|V(s)|_{\Omega}^{2} \mathrm{~d} s$. At the end we return to the energy estimate to deduce that

$$
|\nabla U(t)|_{\Omega}^{2} \leq C_{\varepsilon, \nu, T} \int_{0}^{T}|V(s)|_{\Omega}^{2} \mathrm{~d} s
$$

The continuity of operator $\mathcal{K}$ is then proved from $L^{2}\left(0, T ; \mathbb{L}^{2}(\Omega)\right)$ into $\mathcal{X}$ and consequently operator $\mathcal{K}$ is continuous and compact from $L^{2}\left(0, T ; \mathbb{L}^{2}(\Omega)\right)$ into itself.

Applying Schauder's fixed point theorem, it follows that there exists a fixed point $U_{\nu}^{\varepsilon} \in L^{2}\left(0, T ; \mathbb{L}^{2}(\Omega)\right)$ satisfying $\mathcal{K}\left(U_{\nu}^{\varepsilon}\right)=U_{\nu}^{\varepsilon}$. Moreover setting $\boldsymbol{m}_{\nu}^{\varepsilon}=\mathcal{Q}\left(U_{\nu}^{\varepsilon}\right)$ it follows that $U_{\nu}^{\varepsilon} \in \mathcal{X}, \boldsymbol{m}_{\nu}^{\varepsilon} \in L^{\infty}\left(0, T ; \mathbb{L}^{2}(I)\right) \cap L^{2}\left(0, T ; \mathbb{H}^{1}(I)\right)$ and $\left(U_{\nu}^{\varepsilon}, \boldsymbol{m}_{\nu}^{\varepsilon}\right)$ is a weak solution of (32)-(35).

We proved the following result

Theorem 3.1. Let $\nu>0$ and $\varepsilon>0$ be fixed. Let $\boldsymbol{m}_{0} \in \mathbb{L}^{2}(I), \boldsymbol{M}_{0} \in \mathbb{H}^{1}(\Omega)$ such that $\left|\boldsymbol{M}_{0}(x)\right|^{2}=1$ a.e. and $j_{e} \in L^{2}(\omega)$. Then there exists a weak solution $\left(\boldsymbol{m}_{\nu}^{\varepsilon}, U_{\nu}^{\varepsilon}\right) \in L^{2}\left(0, T ; \mathbb{H}^{1}(I)\right) \times \mathbb{H}^{1}(Q)$ of the coupled problem (32)-(35). Moreover we have $\boldsymbol{m}_{\nu}^{\varepsilon} \in L^{\infty}\left(0, T ; \mathbb{L}^{2}(I)\right), U_{\nu}^{\varepsilon} \in L^{\infty}\left(0, T ; \mathbb{L}^{2}(\Omega)\right)$ and the following energy 
inequalities hold

$$
\begin{aligned}
& \nu^{2}\left|\partial_{t} U_{\nu}^{\varepsilon}(t)\right|_{\Omega}^{2}+\mathcal{E}\left(U_{\nu}^{\varepsilon}(t)\right)+\frac{2}{\varepsilon^{2}} \int_{\Omega} p\left(\left|U_{\nu}^{\varepsilon}(t)\right|\right) \mathrm{d} X \\
& +\frac{\alpha}{\gamma} \int_{0}^{t}\left|\partial_{t} U_{\nu}^{\varepsilon}(s)\right|_{\Omega}^{2} \mathrm{~d} s \leq \mathcal{E}\left(\boldsymbol{M}_{0}\right)+\frac{J^{2}|\widehat{\Omega}| \gamma}{\alpha} \int_{0}^{t}\left|\boldsymbol{m}_{\nu}^{\varepsilon}(s)\right|_{I}^{2} \mathrm{~d} s
\end{aligned}
$$

and

$$
\begin{aligned}
& \frac{1}{2 d^{2}}\left|\boldsymbol{m}_{\nu}^{\varepsilon}(t)\right|_{I}^{2}+\frac{1}{\lambda_{\mathrm{sf}}^{2}} \int_{0}^{t}\left|\boldsymbol{m}_{\nu}^{\varepsilon}(s)\right|_{I}^{2} \mathrm{~d} s+\frac{1-\beta \beta^{\prime}}{2} \int_{0}^{t}\left|\partial_{x} \boldsymbol{m}_{\nu}^{\varepsilon}(s)\right|_{I}^{2} \mathrm{~d} s \\
& \leq \frac{1}{2 d^{2}}\left|\boldsymbol{m}_{0}\right|_{I}^{2}+\frac{\beta^{2}}{2 d^{4}\left(1-\beta \beta^{\prime}\right)} \int_{0}^{T}\left|j_{e}\right|_{I}^{2} \mathrm{~d} s
\end{aligned}
$$

for all $t \in[0, T]$.

\subsection{Passing to the limit for $\nu \rightarrow 0$}

The estimates deduced from (47) and (48) allow to pass to the limit as $\nu \rightarrow 0$ in the coupled problem (32)-(35). We have

Lemma 3.3. There exists $C>0$ which is independent of $\nu$ and $\varepsilon$ such that

$$
\left\{\begin{array}{l}
\left|U_{\nu}^{\varepsilon}\right|_{L^{\infty}\left(0, T ; \mathbb{H}^{1}(\Omega)\right)}+\left|\partial_{t} U_{\nu}^{\varepsilon}\right|_{L^{2}\left(0, T ; \mathbb{L}^{2}(\Omega)\right)}+\left|\nabla \varphi_{\nu}^{\varepsilon}\right|_{L^{\infty}\left(0, T ; \mathbb{L}^{2}\left(\mathbb{R}^{3}\right)\right)} \leq C \\
\left|\boldsymbol{m}_{\nu}^{\varepsilon}\right|_{L^{2}\left(0, T ; \mathbb{H}^{1}(I)\right)}+\left|\partial_{t} \boldsymbol{m}_{\nu}^{\varepsilon}\right|_{L^{2}\left(0, T ; \mathbb{H}^{-1}(I)\right)} \leq C .
\end{array}\right.
$$

The $\mathbb{L}^{2}$-bound of $U_{\nu}^{\varepsilon}$ is deduced from the inequality (associated with the function $p(r))$

$$
\left|U_{\nu}^{\varepsilon}(t)\right|_{\Omega}^{2} \leq|\Omega|+\int_{\Omega} p\left(\left|U_{\nu}^{\varepsilon}(t)\right|\right) \mathrm{d} X+2 \sqrt{2} \int_{\Omega} \sqrt{p\left(\left|U_{\nu}^{\varepsilon}(t)\right|\right)} \mathrm{d} X .
$$

Therefore we can extract subsequences such that $U_{\nu}^{\varepsilon}$ converges towards a limit $U^{\varepsilon}$ weakly in $\mathbb{H}^{1}(Q)$ and $\boldsymbol{m}_{\nu}^{\varepsilon}$ converges to a limit $\boldsymbol{m}^{\varepsilon}$ weakly in $L^{2}\left(0, T ; \mathbb{H}^{1}(I)\right)$. Moreover as a consequence of Aubin's compactness lemma we have the strong convergences

Lemma 3.4. Let $\varepsilon>0$ be fixed. Under the hypotheses of Theorem 3.1 there exist subsequences $U_{\nu}^{\varepsilon}$ and $\boldsymbol{m}_{\nu}^{\varepsilon}$ such that

$$
\left\{\begin{array}{l}
U_{\nu}^{\varepsilon} \rightarrow U^{\varepsilon} \text { strongly in } L^{2}\left(0, T ; \mathbb{L}^{2}(\Omega)\right) \\
\rho^{\nu} \star \widetilde{U}_{\nu}^{\varepsilon} \rightarrow \widetilde{U}^{\varepsilon} \text { strongly in } \mathbb{L}^{2}\left(\mathbb{R}^{4}\right) \\
\boldsymbol{m}_{\nu}^{\varepsilon} \rightarrow \boldsymbol{m}^{\varepsilon} \text { strongly in } L^{2}\left(0, T ; \mathbb{L}^{2}(I)\right) .
\end{array}\right.
$$

Since $\mathcal{S}^{\varepsilon}$ is Lipschitz continuous, we get that $\mathcal{S}^{\varepsilon}\left(\rho^{\nu} \star \widetilde{U}_{\nu}^{\varepsilon}\right) \rightarrow \mathcal{S}^{\varepsilon}\left(U^{\varepsilon}\right)$ strongly in $\mathbb{L}^{2}(Q)$ which allows to pass to the limit in (32). Therefore the equation (23) is satisfied in the sense of distributions and the boundary conditions are fulfilled. Moreover the property of $\mathcal{T}^{\varepsilon}$ leads to the weak convergence

$$
\mathcal{A}^{\varepsilon}\left(\left\langle\rho^{\nu} \star \widetilde{U}_{\nu}^{\varepsilon}\right\rangle\right)\left(\partial_{x} \boldsymbol{m}_{\nu}^{\varepsilon}\right) \rightarrow \mathcal{A}^{\varepsilon}\left(\left\langle U^{\varepsilon}\right\rangle\right)\left(\partial_{x} \boldsymbol{m}^{\varepsilon}\right) \text { in } \mathbb{L}^{2}(\omega)
$$


as well as the strong convergences

$$
\boldsymbol{m}_{\nu}^{\varepsilon} \times \mathcal{T}^{\varepsilon}\left(\rho^{\nu} \star \widetilde{U}_{\nu}^{\varepsilon}\right) \rightarrow \boldsymbol{m}^{\varepsilon} \times \mathcal{T}^{\varepsilon}\left(U^{\varepsilon}\right) \text { in } \mathbb{L}^{2}(\omega)
$$

and

$$
j_{e} \mathcal{T}^{\varepsilon}\left(\rho^{\nu} \star \widetilde{U}_{\nu}^{\varepsilon}\right) \rightarrow j_{e} \mathcal{T}^{\varepsilon}\left(U^{\varepsilon}\right) \text { in } \mathbb{L}^{2}(\omega)
$$

Thus $\boldsymbol{m}^{\varepsilon}$ satisfies in the sense of distributions the equation

$$
\begin{aligned}
\frac{1}{d^{2}} \partial_{t} \boldsymbol{m}^{\varepsilon}-\partial_{x}\left(\mathcal{A}^{\varepsilon}\left(\left\langle U^{\varepsilon}\right\rangle\right)\left(\partial_{x} \boldsymbol{m}^{\varepsilon}\right)\right)+\frac{1}{\lambda_{\mathrm{sf}}^{2}} \boldsymbol{m}^{\varepsilon} \\
=-\frac{1}{\lambda_{J}^{2}} \boldsymbol{m}^{\varepsilon} \times \mathcal{T}^{\varepsilon}\left(U^{\varepsilon}\right)-\frac{\beta}{d^{2}} \partial_{x}\left(j_{e} \mathcal{T}^{\varepsilon}\left(U^{\varepsilon}\right)\right) .
\end{aligned}
$$

Consequently $\left(1 / d^{2}\right) \partial_{t} \boldsymbol{m}^{\varepsilon}-\partial_{x}\left(\mathcal{A}^{\varepsilon}\left(\left\langle U^{\varepsilon}\right\rangle\right)\left(\partial_{x} \boldsymbol{m}^{\varepsilon}\right)-\left(\beta / d^{2}\right) j_{e} \mathcal{T}^{\varepsilon}\left(U^{\varepsilon}\right)\right) \in \mathbb{L}^{2}(\omega)$ so as if we set $p^{\varepsilon}=-\mathcal{A}^{\varepsilon}\left(\left\langle U^{\varepsilon}\right\rangle\right)\left(\partial_{x} \boldsymbol{m}^{\varepsilon}\right)+\left(\beta / d^{2}\right) j_{e} \mathcal{T}^{\varepsilon}\left(U^{\varepsilon}\right)$, we have $\left.\left(\left(1 / d^{2}\right) \boldsymbol{m}^{\varepsilon}, p^{\varepsilon}\right) \cdot N\right|_{\partial \omega}$ which is well defined in $\mathbb{H}^{-1 / 2}(\partial \omega)$. Here $N$ denotes the outward unit normal to the boundary $\partial \omega$ of $\omega$.

Let $\theta \in \mathbb{H}^{1 / 2}(\partial \omega)$ and $\eta \in \mathbb{H}^{1}(\omega)$ such that $\left.\eta\right|_{\partial \omega}=\theta$. Thus setting $p_{\nu}^{\varepsilon}=$ $-\mathcal{A}^{\varepsilon}\left(\left\langle\rho^{\nu} \star \widetilde{U}_{\nu}^{\varepsilon}\right\rangle\right)\left(\partial_{x} \boldsymbol{m}_{\nu}^{\varepsilon}\right)+\left(\beta / d^{2}\right) j_{e} \mathcal{T}^{\varepsilon}\left(\rho^{\nu} \star \widetilde{U}_{\nu}^{\varepsilon}\right)$ and $D_{\nu}^{\varepsilon}=\left(\left(1 / d^{2}\right)\left(\boldsymbol{m}_{\nu}^{\varepsilon}-\boldsymbol{m}^{\varepsilon}\right), p_{\nu}^{\varepsilon}-p^{\varepsilon}\right)$ we have

$$
\int_{\partial \omega} \theta D_{\nu}^{\varepsilon} \cdot N d \sigma=\int_{\omega} \nabla \eta \cdot D_{\nu}^{\varepsilon} \mathrm{d} x \mathrm{~d} t+\int_{\omega} \eta \nabla \cdot D_{\nu}^{\varepsilon} \mathrm{d} x \mathrm{~d} t
$$

where $\mathrm{d} \sigma$ is the Lebesgue measure on $\partial \omega, \nabla=\left(\partial_{t}, \partial_{x}\right)$. We get

$$
\begin{aligned}
\int_{\partial \omega} \theta D_{\nu}^{\varepsilon} \cdot N d \sigma= & \int_{\omega} \nabla \eta \cdot D_{\nu}^{\varepsilon} \mathrm{d} x \mathrm{~d} t-\left(1 / \lambda_{\mathrm{sf}}^{2}\right) \int_{\omega} \eta\left(\boldsymbol{m}_{\nu}^{\varepsilon}-\boldsymbol{m}^{\varepsilon}\right) \mathrm{d} x \mathrm{~d} t \\
& -\left(1 / \lambda_{J}^{2}\right) \int_{\omega} \eta\left(\boldsymbol{m}_{\nu}^{\varepsilon} \times \mathcal{T}^{\varepsilon}\left(\rho^{\nu} \star \widetilde{U}_{\nu}^{\varepsilon}\right)-\boldsymbol{m}^{\varepsilon} \times \mathcal{T}^{\varepsilon}\left(U^{\varepsilon}\right)\right) \mathrm{d} x \mathrm{~d} t
\end{aligned}
$$

which leads to $\lim _{\nu \rightarrow 0} \int_{\partial \omega} \theta D_{\nu}^{\varepsilon} \cdot N \mathrm{~d} \sigma=0$ that is

$$
\begin{aligned}
& \lim _{\nu \rightarrow 0}\left(\left(1 / d^{2}\right)\left(\int_{I} \theta(T, x)\left(\boldsymbol{m}_{\nu}^{\varepsilon}-\boldsymbol{m}^{\varepsilon}\right)(T, x) \mathrm{d} x-\int_{I} \theta(0, x)\left(\boldsymbol{m}_{0}(x)-\boldsymbol{m}^{\varepsilon}(0, x)\right) \mathrm{d} x\right)\right. \\
& \left.\quad+\int_{0}^{T} \theta(t, l)\left(p_{\nu}^{\varepsilon}-p^{\varepsilon}\right)(t, l) \mathrm{d} t-\int_{0}^{T} \theta(t,-L)\left(p_{\nu}^{\varepsilon}-p^{\varepsilon}\right)(t,-L) \mathrm{d} t\right)=0
\end{aligned}
$$

Then for adequate test functions $\theta$, we get the desired boundary conditions. So $\boldsymbol{m}^{\varepsilon}$ satisfies (24). We proved the following result

TheOrem 3.2. Let $\varepsilon>0$ be fixed. Let $\boldsymbol{m}_{0} \in \mathbb{L}^{2}(I), \boldsymbol{M}_{0} \in \mathbb{H}^{1}(\Omega)$ such that $\left|\boldsymbol{M}_{0}(X)\right|^{2}=1$ a.e. and $j_{e} \in L^{2}(\omega)$. Then there exists a weak solution $\left(\boldsymbol{m}^{\varepsilon}, U^{\varepsilon}\right) \in$ $L^{2}\left(0, T ; \mathbb{H}^{1}(I)\right) \times \mathbb{H}^{1}(Q)$ of the coupled problem (23)-(24). Moreover we have $\boldsymbol{m}^{\varepsilon} \in$ 
$L^{\infty}\left(0, T ; \mathbb{L}^{2}(I)\right), U^{\varepsilon} \in L^{\infty}\left(0, T ; \mathbb{H}^{1}(\Omega)\right)$ and the following energy inequalities hold

$$
\begin{aligned}
& \left|\sqrt{a} \nabla U^{\varepsilon}(t)\right|_{\Omega}^{2}+\frac{2}{\varepsilon^{2}} \int_{\Omega} p\left(\left|U^{\varepsilon}(t)\right|\right) \mathrm{d} X \\
& \quad+\left|\nabla \varphi^{\varepsilon}(t)\right|_{\mathbb{R}^{3}}^{2}+2 \int_{\Omega} \psi\left(U^{\varepsilon}(t)\right) \mathrm{d} X+\frac{\alpha}{\gamma} \int_{0}^{t}\left|\partial_{t} U^{\varepsilon}(s)\right|_{\Omega}^{2} \mathrm{~d} s \\
& \leq \mathcal{E}\left(\boldsymbol{M}_{0}\right)+\frac{J^{2}|\widehat{\Omega}| \gamma}{\alpha} \int_{0}^{t}\left|\boldsymbol{m}^{\varepsilon}(s)\right|_{I}^{2} \mathrm{~d} s
\end{aligned}
$$

and

$$
\begin{aligned}
& \frac{1}{2 d^{2}}\left|\boldsymbol{m}^{\varepsilon}(t)\right|_{I}^{2}+\frac{1}{\lambda_{\mathrm{sf}}^{2}} \int_{0}^{t}\left|\boldsymbol{m}^{\varepsilon}(s)\right|_{I}^{2} \mathrm{~d} s+\frac{1-\beta \beta^{\prime}}{2} \int_{0}^{t}\left|\partial_{x} \boldsymbol{m}^{\varepsilon}(s)\right|_{I}^{2} \mathrm{~d} s \\
& \leq \frac{1}{2 d^{2}}\left|\boldsymbol{m}_{0}\right|_{I}^{2}+\frac{\beta^{2}}{2 d^{4}\left(1-\beta \beta^{\prime}\right)} \int_{0}^{T}\left|j_{e}\right|_{I}^{2} \mathrm{~d} s
\end{aligned}
$$

for $t \in[0, T]$.

\subsection{Global solutions to (8)-(3)}

We want to pass to the limit as $\varepsilon \rightarrow 0$ in the system (23)-(24). The estimate satisfied by $U^{\varepsilon}$ given in Theorem 3.2 is rewritten as

$$
\begin{aligned}
& \mathcal{E}^{\varepsilon}\left(U^{\varepsilon}(t)\right)+\frac{\alpha}{\gamma} \int_{0}^{t}\left|\partial_{t} U^{\varepsilon}\right|_{\Omega} \mathrm{d} s+\frac{2}{\varepsilon^{2}} \int_{\Omega} p\left(\left|U^{\varepsilon}(t)\right|\right) \mathrm{d} X \\
& \leq \mathcal{E}\left(\boldsymbol{M}_{0}\right)+C_{0}\left|\boldsymbol{m}_{0}\right|_{I}^{2}+C_{1} \int_{0}^{T}\left|j_{e}\right|_{I}^{2} \mathrm{~d} s
\end{aligned}
$$

where $C_{0}>0$ and $C_{1}>0$ are two positive constants which are independent of $\varepsilon$ and $T$. It follows that

$$
\int_{\Omega} p\left(\left|U^{\varepsilon}(t)\right|\right) \mathrm{d} X \leq C \varepsilon^{2}
$$

Since we have $r^{2}-1=p(r)+2 \sqrt{2}\left(\sqrt{1+r^{2}}-\sqrt{2}\right)$ then we get

$$
\left.\int_{\Omega}|| U^{\varepsilon}\right|^{2}-1 \mid \mathrm{d} X \leq C\left(\varepsilon^{2}+|\Omega|^{1 / 2} \varepsilon\right) .
$$

Hence the sequence $\left(U^{\varepsilon}\right)_{\varepsilon}$ is bounded in $L^{\infty}\left(0, T ; \mathbb{L}^{2}(\Omega)\right)$. It follows that there exists a subsequence and $M \in \mathbb{L}^{2}(Q)$ such that we have

$$
\left\{\begin{array}{l}
U^{\varepsilon} \rightarrow M \text { weakly in } \mathbb{L}^{2}(Q) \\
\left|U^{\varepsilon}\right|^{2} \rightarrow 1 \text { strongly in } \mathbb{L}^{1}(Q) .
\end{array}\right.
$$

The energy inequality leads also to

$$
\left|\partial_{t} U^{\varepsilon}\right|_{L^{2}\left(0, T ; \mathbb{L}^{2}(\Omega)\right)}+\left|\nabla U^{\varepsilon}\right|_{L^{\infty}\left(0, T ; \mathbb{L}^{2}(\Omega)\right)} \leq C
$$


which implies by using the classical compactness lemma the strong convergence

$$
U^{\varepsilon} \rightarrow \boldsymbol{M} \text { strongly in } L^{2}\left(0, T ; \mathbb{L}^{2}(\Omega)\right)
$$

so we have

$$
|\boldsymbol{M}(t, X)|^{2}=1 \text { a.e. in } Q .
$$

We pass to the limit in the weak formulation of the intermediary problem (23) satisfied by $U^{\varepsilon}$. We use test functions of the type $\phi \times U^{\varepsilon}$ where $\phi$ is a test function defined in $Q$ satisfying $\phi(t, h+0, \widehat{x})=\phi(t,-h-0, \widehat{x})$ in $(0, T) \times \widehat{\Omega}$. We have

$$
\begin{aligned}
& \frac{\alpha}{\gamma} \int_{Q} \partial_{t} U^{\varepsilon} \cdot \phi \times U^{\varepsilon} \mathrm{d} X \mathrm{~d} t+\int_{Q} a \nabla U^{\varepsilon} \cdot \nabla \phi \times U^{\varepsilon} \mathrm{d} X \mathrm{~d} t \\
& \quad-\int_{Q} \mathcal{R}^{\varepsilon}\left(U^{\varepsilon}, \partial_{t} U^{\varepsilon}\right) \cdot \phi \times U^{\varepsilon} \mathrm{d} X \mathrm{~d} t \\
& =\int_{Q} \mathcal{L}\left(U^{\varepsilon}\right) \cdot \phi \times U^{\varepsilon} \mathrm{d} X \mathrm{~d} t+J \int_{Q} \boldsymbol{m}^{\varepsilon} \cdot \phi \times U^{\varepsilon} \mathrm{d} X \mathrm{~d} t .
\end{aligned}
$$

We get the following result

Theorem 3.3. Let $T>0$ be fixed and $\boldsymbol{M}_{0} \in \mathbb{H}^{1}(\Omega)$ be such that $\left|\boldsymbol{M}_{0}(X)\right|^{2}=$ 1 a.e., $\boldsymbol{m}_{0} \in \mathbb{L}^{2}(I)$ and $j_{e} \in L^{2}(\omega)$. Then, there exists a global weak solution $(\boldsymbol{m}, \boldsymbol{M})$ of the coupled problem (8)-(12)-(3)-(11) such that $\boldsymbol{M} \in L^{\infty}(Q) \cap \mathbb{H}^{1}(Q)$, $|\boldsymbol{M}(t, X)|^{2}=1$ a.e., $\boldsymbol{m} \in L^{\infty}\left(0, T ; \mathbb{L}^{2}(I)\right) \cap L^{2}\left(0, T ; \mathbb{H}^{1}(I)\right)$ and satisfying the energy inequalities

$$
\begin{gathered}
\mathcal{E}(\boldsymbol{M}(t))+\frac{\alpha}{\gamma} \int_{0}^{t}\left|\partial_{t} \boldsymbol{M}(s)\right|_{\Omega}^{2} \mathrm{~d} s \leq \mathcal{E}\left(\boldsymbol{M}_{0}\right)+C_{0}\left|\boldsymbol{m}_{0}\right|_{I}^{2}+C_{1} \int_{0}^{T}\left|j_{e}\right|_{I}^{2} \mathrm{~d} s \\
|\boldsymbol{m}(t)|_{I}^{2}+C_{2} \int_{0}^{t}|\boldsymbol{m}(s)|_{I}^{2} \mathrm{~d} s+\frac{1-\beta \beta^{\prime}}{2} \int_{0}^{t}\left|\partial_{x} \boldsymbol{m}(s)\right|_{I}^{2} \mathrm{~d} s \\
\leq C_{0}\left|\boldsymbol{m}_{0}\right|_{I}^{2}+C_{1} \int_{0}^{T}\left|j_{e}\right|_{I}^{2} \mathrm{~d} s
\end{gathered}
$$

for all $t \in[0, T]$ where $C_{0}>0, C_{1}$ and $C_{2}$ are positive constants independent of $(\boldsymbol{m}, \boldsymbol{M})$ and $T$.

Proof. It is easy to see that the operator $M \mapsto \nabla \varphi$ with $\nabla \cdot(\nabla \varphi+\chi(\Omega) M)=0$ in $(0, T) \times \mathbb{R}^{3}$ is linear continuous from $\mathbb{L}^{2}$ into itself. Therefore we can pass to the limit in the stray field equation by using the strong convegence of $U^{\varepsilon}$ to obtain that the strong limit $\nabla \varphi$ of $\nabla \varphi^{\varepsilon}$ in $\mathbb{L}^{2}\left((0, T) \times \mathbb{R}^{3}\right)$ satisfies the stray field equation (10). Moreover the Lipschitz continuity of $\nabla_{U} \psi$ allows to pass to the limit in the volume anisotropy field $\nabla_{U} \psi\left(U^{\varepsilon}\right)$ so that we have $\mathcal{L}\left(U^{\varepsilon}\right) \rightarrow \mathcal{L}(\boldsymbol{M})$ strongly in $L^{2}\left(0, T ; \mathbb{L}^{2}(\Omega)\right)$. Next, for all $\phi \in \mathbb{L}^{2}(Q)$ we have $\mathcal{S}^{\varepsilon}\left(U^{\varepsilon}\right) \times \phi \rightarrow \boldsymbol{M} \times \phi$ a.e. and strongly in $L^{2}\left(0, T ; \mathbb{L}^{2}(\Omega)\right)$. Consequently $\mathcal{R}^{\varepsilon}\left(U^{\varepsilon}, \partial_{t} U^{\varepsilon}\right) \rightarrow \frac{1}{\gamma} \boldsymbol{M} \times \partial_{t} \boldsymbol{M}$ weakly in $L^{2}\left(0, T ; \mathbb{L}^{2}(\Omega)\right)$ and then $\int_{Q} \mathcal{R}^{\varepsilon}\left(U^{\varepsilon}, \partial_{t} U^{\varepsilon}\right) \cdot \phi \times U^{\varepsilon} \mathrm{d} X \mathrm{~d} t \rightarrow \frac{1}{\gamma} \int_{Q} \boldsymbol{M} \times \partial_{t} \boldsymbol{M} \cdot \phi \times$ 
$M \mathrm{~d} X \mathrm{~d} t$. We pass to the limit in (65) to get

$$
\begin{aligned}
& \frac{\alpha}{\gamma} \int_{Q} \partial_{t} \boldsymbol{M} \cdot \phi \times \boldsymbol{M} \mathrm{d} X \mathrm{~d} t+\int_{Q} a(X) \nabla \boldsymbol{M} \cdot \nabla \phi \times \boldsymbol{M} \mathrm{d} X \mathrm{~d} t \\
& \quad-\frac{1}{\gamma} \int_{Q} \boldsymbol{M} \times \partial_{t} \boldsymbol{M} \cdot \phi \times \boldsymbol{M} \mathrm{d} X \mathrm{~d} t \\
& =\int_{Q} \mathcal{L}(\boldsymbol{M}) \cdot \phi \times \boldsymbol{M} \mathrm{d} X \mathrm{~d} t+J \int_{Q} \boldsymbol{m} \cdot \phi \times \boldsymbol{M} \mathrm{d} X \mathrm{~d} t
\end{aligned}
$$

where $\boldsymbol{m}$ is the weak limit of $\boldsymbol{m}^{\varepsilon}$ in $L^{2}\left(0, T ; \mathbb{L}^{2}(I)\right)$. Hence $\boldsymbol{M}$ satisfies the equation (8) with the initial-boundary conditions (12). In addition of the energy inequality, $\boldsymbol{m}^{\varepsilon}$ is such that $\left(\partial_{t} \boldsymbol{m}^{\varepsilon}\right)_{\varepsilon}$ is uniformly bounded in $L^{2}\left(0, T ; \mathbb{H}^{-1}(I)\right)$ so $\boldsymbol{m}^{\varepsilon}$ converges towards $\boldsymbol{m}$ weakly in $L^{2}\left(0, T ; \mathbb{H}^{1}(I)\right)$ and strongly in $L^{2}\left(0, T ; \mathbb{L}^{2}(I)\right)$. Let us consider the convergence of $\mathcal{A}^{\varepsilon}\left(\left\langle U^{\varepsilon}\right\rangle\right)\left(\partial_{x} \boldsymbol{m}^{\varepsilon}\right)$. First of all $\left\langle U^{\varepsilon}\right\rangle \rightarrow\langle\boldsymbol{M}\rangle,\left\langle\left|U^{\varepsilon}\right|\right\rangle \rightarrow 1$ a.e. in $\omega$. Therefore $\mathcal{T}^{\varepsilon}\left(U^{\varepsilon}\right) \rightarrow\langle\boldsymbol{M}\rangle$ and $\mathcal{T}^{\varepsilon}\left(U^{\varepsilon}\right) \otimes \mathcal{T}^{\varepsilon}\left(U^{\varepsilon}\right) \rightarrow\langle\boldsymbol{M}\rangle \otimes\langle\boldsymbol{M}\rangle$ a.e. in $\omega$ so $\mathcal{T}^{\varepsilon}\left(U^{\varepsilon}\right) \otimes \mathcal{T}^{\varepsilon}\left(U^{\varepsilon}\right) \phi \rightarrow\langle\boldsymbol{M}\rangle \otimes\langle\boldsymbol{M}\rangle \phi$ strongly in $\mathbb{L}^{2}(\omega)$ for all $\phi \in \mathbb{L}^{2}(\omega)$. Consequently, we obtain

$$
\mathcal{A}^{\varepsilon}\left(\left\langle U^{\varepsilon}\right\rangle\right)\left(\partial_{x} \boldsymbol{m}^{\varepsilon}\right) \rightarrow \mathcal{A}(\langle\boldsymbol{M}\rangle)\left(\partial_{x} \boldsymbol{m}\right) \text { weakly in } \mathbb{L}^{2}(\omega) .
$$

A similar argument gives $\boldsymbol{m}^{\varepsilon} \times \mathcal{T}^{\varepsilon}\left(U^{\varepsilon}\right) \rightarrow \boldsymbol{m} \times\langle\boldsymbol{M}\rangle$ weakly in $\mathbb{L}^{2}(\omega)$ and $j_{e} \mathcal{T}^{\varepsilon}\left(U^{\varepsilon}\right) \rightarrow$ $j_{e}\langle\boldsymbol{M}\rangle$ strongly in $\mathbb{L}^{2}(\omega)$ so taking the limit in (24), we get the equation (3). Proceeding along the lines of the precedent paragraph, we verify that $\boldsymbol{m}$ satisfies the initial and boundary conditions (11).

Finally we consider the energy inequalities satisfied by $U^{\varepsilon}$ and $\boldsymbol{m}^{\varepsilon}$, we make use of the weak and strong convergences obtained and the lower semi-continuity of the norms to deduce the wished energy estimates on $\boldsymbol{M}$ and $\boldsymbol{m}$.

\section{The Stationary Spin Accumulation Equation}

The time scale appearing in the spin magnetization is shorter than the time scale of the LLG equation (see [19], [11] for a discussion) then we may consider stationary solutions of the spin accumulation equation

$$
\left\{\begin{array}{l}
\partial_{x}\left(\mathcal{A}(\langle\boldsymbol{M}\rangle) \partial_{x} \boldsymbol{m}\right)-\frac{1}{\lambda_{\mathrm{sf}}^{2}} \boldsymbol{m}-\frac{1}{\lambda_{J}^{2}} \boldsymbol{m} \times\langle\boldsymbol{M}\rangle=\frac{\beta}{d^{2}} \partial_{x}\left(j_{e}\langle\boldsymbol{M}\rangle\right) \\
\boldsymbol{j}_{m}(t,-L)=\boldsymbol{j}_{m}(t, l)=0
\end{array}\right.
$$

where $j_{e}$ is assumed to be independent of time. We are interested with the global solutions to the system (8)-(12)-(68). We should verify that the energy associated with $\boldsymbol{m}$ allows to deduce an uniform bound for the energy of $\boldsymbol{M}$. For all $t \geq 0$, we have

$$
\frac{\left(1-\beta \beta^{\prime}\right)}{2}\left|\partial_{x} \boldsymbol{m}\right|_{I}^{2}+\frac{1}{\lambda_{\mathrm{sf}}^{2}}|\boldsymbol{m}|_{I}^{2} \leq \frac{\beta^{2}}{2 d^{4}\left(1-\beta \beta^{\prime}\right)}\left|j_{e}\right|_{I}^{2}
$$


Next, it holds that

$$
\begin{aligned}
& J\left|\int_{0}^{t} \int_{\Omega} \boldsymbol{m} \cdot \partial_{t} \boldsymbol{M} \mathrm{d} X \mathrm{~d} s\right| \\
& \quad \leq \frac{\alpha}{2 \gamma} \int_{0}^{t} \int_{\Omega}\left|\partial_{t} \boldsymbol{M}\right|^{2} \mathrm{~d} X \mathrm{~d} s+\frac{J^{2} \gamma|\widehat{\Omega}|}{2 \alpha} \frac{\beta^{2} \lambda_{\mathrm{sf}}^{2}}{2 d^{4}\left(1-\beta \beta^{\prime}\right)}\left|j_{e}\right|_{I}^{2} t
\end{aligned}
$$

Hence our method allows to prove a global existence result for weak solutions to the system (8)-(12)-(68). To do that we introduce the regularized problem

$$
\left\{\begin{array}{l}
\nu^{2} \partial_{t}^{2} U_{\nu}^{\varepsilon}-\nabla \cdot\left(a(X) \nabla U_{\nu}^{\varepsilon}\right)+\mathcal{B}^{\varepsilon}\left(\rho^{\nu} \star \widetilde{U}_{\nu}^{\varepsilon}, \partial_{t} U_{\nu}^{\varepsilon}\right) \\
\quad=-\frac{1}{\varepsilon^{2}} \nabla_{U}\left(p\left(\left|U_{\nu}^{\varepsilon}\right|\right)\right)+\mathcal{L}\left(U_{\nu}^{\varepsilon}\right)+J \boldsymbol{m}_{\nu}^{\varepsilon} \\
U_{\nu}^{\varepsilon}(0, X)=\boldsymbol{M}_{0}(X),\left|\boldsymbol{M}_{0}(X)\right|^{2}=1 \text { a.e., } \nu^{2} \partial_{t} U_{\nu}^{\varepsilon}(0, X)=0 \text { in } \Omega
\end{array}\right.
$$

coupled to the elliptic equation

$$
\begin{aligned}
&- \partial_{x}\left(\mathcal{A}^{\varepsilon}\left(\left\langle\rho^{\nu} \star \widetilde{U}_{\nu}^{\varepsilon}\right\rangle\right)\left(\partial_{x} \boldsymbol{m}_{\nu}^{\varepsilon}\right)\right)+\frac{1}{\lambda_{\mathrm{sf}}^{2}} \boldsymbol{m}_{\nu}^{\varepsilon} \\
&=-\frac{1}{\lambda_{J}^{2}} \boldsymbol{m}_{\nu}^{\varepsilon} \times \mathcal{T}^{\varepsilon}\left(\rho^{\nu} \star \widetilde{U}_{\nu}^{\varepsilon}\right)-\frac{\beta}{d^{2}} \partial_{x}\left(j_{e} \mathcal{T}^{\varepsilon}\left(\rho^{\nu} \star \widetilde{U}_{\nu}^{\varepsilon}\right)\right)
\end{aligned}
$$

with the boundary conditions defined previously. Proceeding along the lines of the proof given in Section 3 we can prove the same global existence result of weak solutions $(\boldsymbol{m}, \boldsymbol{M})$ on the finite time interval $[0, T]$ for all $T>0$ fixed. More precisely, we have

Proposition 4.1. Let $T>0$ be fixed, $\boldsymbol{M}_{0} \in \mathbb{H}^{1}(\Omega)$ be such that $\left|\boldsymbol{M}_{0}(X)\right|^{2}=$ 1 a.e. and $j_{e} \in L^{2}(I)$. There exists a global weak solution $(\boldsymbol{m}, \boldsymbol{M})$ of the coupled system (8)-(12)-(68) such that $\boldsymbol{M} \in \mathbb{L}^{\infty}(Q) \cap \mathbb{H}^{1}(Q),|\boldsymbol{M}(t, X)|^{2}=1$ a.e., $\boldsymbol{m} \in$ $\mathbb{H}^{1}(I)$ and satisfying the energy inequalities

$$
\begin{gathered}
\mathcal{E}(\boldsymbol{M}(t))+\frac{\alpha}{\gamma} \int_{0}^{t}\left|\partial_{t} \boldsymbol{M}(s)\right|_{\Omega}^{2} \mathrm{~d} s \leq \mathcal{E}\left(\boldsymbol{M}_{0}\right)+C_{1} T\left|j_{e}\right|_{I}^{2}, \\
\frac{1}{\lambda_{s f}^{2}}|\boldsymbol{m}|_{I}^{2}+\frac{1-\beta \beta^{\prime}}{2}\left|\partial_{x} \boldsymbol{m}\right|_{I}^{2} \leq C_{2}\left|j_{e}\right|_{I}^{2}
\end{gathered}
$$

for all $t \in[0, T]$ where $C_{1}>0$ and $C_{2}$ are positive constants which are independent of $(\boldsymbol{m}, \boldsymbol{M})$ and $T$.

REMARK 4.1. The energy estimate shows that the energy satisfies the upper bound $\mathcal{E}(\boldsymbol{M}(t)) \leq C_{0}+C_{1} T$. It is interesting to obtain a lower bound of that energy.

\section{Concluding Remarks}

In the case where the local magnetization is uniform in the ferromagnet layer the torque $\boldsymbol{m}_{\perp}$ acting on the switching of the magnetization takes the form see [19] 
and $[11]$

$$
J \boldsymbol{m}_{\perp}=b M^{2}+a M^{2} \times M^{1}
$$

where the transverse spin accumulation $\boldsymbol{m}_{\perp}$ represents the components of $\boldsymbol{m}$ which are orthogonal to $\boldsymbol{M}^{1}$, the local magnetization of the thin layer $\Omega^{1}, a$ and $b$ are two constants. We denote by $\boldsymbol{M}^{2}$ the local magnetization of the thick layer $\Omega^{2}$. It is assumed that in the thick ferromagnet, the local magnetization $\boldsymbol{M}^{2}$ polarizes the current and is uniform and pinned. Notice that the longitudinal component $\boldsymbol{m}_{\|}$ (parallel to $\boldsymbol{M}^{1}$ ) of the spin accumulation has no effect in the product $\boldsymbol{M}^{1} \times \boldsymbol{m}$. Hence, the LLG equation becomes

$$
\partial_{t} \boldsymbol{M}-\alpha \boldsymbol{M} \times \partial_{t} \boldsymbol{M}=-\gamma \boldsymbol{M} \times\left(\mathcal{H}_{e}+b \boldsymbol{V}+a \boldsymbol{V} \times \boldsymbol{M}\right)
$$

where we set $\boldsymbol{M}=\boldsymbol{M}^{1}$ and $\boldsymbol{V}=\boldsymbol{M}^{2}$. Generally, the following form $\boldsymbol{V}=\cos (\theta) \boldsymbol{e}_{3}-$ $\sin (\theta) \boldsymbol{e}_{1}$ is used where $\theta \in[0, \pi / 2]$ is the angle between $\boldsymbol{V}$ and $\boldsymbol{e}_{3}$ the final position of $\boldsymbol{M}$. In this model the effective magnetization field does not contain the exchange magnetic field $\nabla \cdot(a \nabla \boldsymbol{M})$ and then the LLG becomes a set of ordinary differential equations.

In [15], the authors developped a theory of domain wall dynamics including the effect of electric current in the two limiting cases: adiabatic (thick) and thin walls. They also presented some expectations based on qualitative behaviour. In order to see whether this experimental situation can be understood quantitatively, a micromagnetic study is proposed in [17]. Let us discuss the model used in [17] to describe the switching of the local magnetization across a ferromagnetic domain wall. Let $\Omega=(-l, l) \times \widehat{\Omega}$ be a bounded and regular horizontal cylinder of $\mathbb{R}^{3}$ representing the ferromagnet. The cross section $\widehat{\Omega}$ is included in the plane $\left(\boldsymbol{e}_{2}, \boldsymbol{e}_{3}\right)$. The local magnetization satisfies in $\Omega$ the equation

$$
\partial_{t} \boldsymbol{M}-\alpha \boldsymbol{M} \times \partial_{t} \boldsymbol{M}=-\gamma \boldsymbol{M} \times \mathcal{H}_{e}-u \partial_{x} \boldsymbol{M}
$$

where $x$ denotes the coordinate along which the current flows, $X=(x, \widehat{x}) \in \Omega$ with $\widehat{x}=(y, z)$. The effective magnetic field $\mathcal{H}_{e}$ contains exchange energy, volume anisotropy energy and magnetostatic. The initial-boundary conditions are

$$
\boldsymbol{M}(0, X)=\boldsymbol{M}_{0}(X) \text { in } \Omega, \quad \boldsymbol{M} \times((a \nabla \cdot n) \boldsymbol{M})=0 \text { on }(0, T) \times \partial \Omega .
$$

Multiplying equation (76) by $\boldsymbol{M}$, we get the transport equation $\partial_{t}|\boldsymbol{M}|^{2}+u \partial_{x}|\boldsymbol{M}|^{2}=$ 0 which implies the saturation condition if initially it holds. Therefore we may write $u \partial_{x} \boldsymbol{M}=-u \boldsymbol{M} \times\left(\boldsymbol{M} \times \partial_{x} \boldsymbol{M}\right)$. The equation (76) has the equivalent form

$$
\partial_{t} \boldsymbol{M}-\alpha \boldsymbol{M} \times \partial_{t} \boldsymbol{M}=-\gamma \boldsymbol{M} \times\left(\mathcal{H}_{e}-\frac{u}{\gamma} \boldsymbol{M} \times \partial_{x} \boldsymbol{M}\right)
$$

A model equations of the same type is used in [1] to describe the dynamic of the local magnetization in the presence of spin-polarized current in a layer of the 
type $F / N / F$. The modified LLG equations in the ferromagnet layer $\Omega$ are given by

$$
\partial_{t} \boldsymbol{M}-\alpha \boldsymbol{M} \times \partial_{t} \boldsymbol{M}=-\gamma \boldsymbol{M} \times\left(\mathcal{H}_{e}+\beta \boldsymbol{M} \times(\boldsymbol{j} \cdot \nabla) \boldsymbol{M}\right)
$$

where $\beta>0$ is a constant and $j$ the current. Initial-boundary conditions and transmission boundary conditions at the interfaces $F / N$ and $N / F$ are assumed to be verified. The equations can be rewritten as

$$
\partial_{t} \boldsymbol{M}-\alpha \boldsymbol{M} \times \partial_{t} \boldsymbol{M}=-\gamma \boldsymbol{M} \times\left(\mathcal{H}_{e}-\beta(\boldsymbol{j} \cdot \nabla) \boldsymbol{M}\right) .
$$

To solve this problem, we proceed as in Section 3 and introduce the regularizedpenalized equations

$$
\left\{\begin{array}{c}
\nu^{2} \partial_{t}^{2} U_{\nu}^{\varepsilon}-\nabla \cdot\left(a \nabla U_{\nu}^{\varepsilon}\right)+\mathcal{B}^{\varepsilon}\left(\rho^{\nu} \star \widetilde{U}_{\nu}^{\varepsilon}, \partial_{t} U_{\nu}^{\varepsilon}\right) \\
=-\frac{1}{\varepsilon^{2}} \nabla_{U}\left(p\left(\left|U_{\nu}^{\varepsilon}\right|\right)\right)+\mathcal{L}\left(U_{\nu}^{\varepsilon}\right)+\beta(\boldsymbol{j} \cdot \nabla) U_{\nu}^{\varepsilon} \\
U_{\nu}^{\varepsilon}(0, X)=\boldsymbol{M}_{0}(X),\left|\boldsymbol{M}_{0}(X)\right|^{2}=1 \text { a.e., } \nu^{2} \partial_{t} U_{\nu}^{\varepsilon}(0, X)=0 .
\end{array}\right.
$$

The following energy estimate holds

$$
\begin{aligned}
& \nu^{2}\left|\partial_{t} U_{\nu}^{\varepsilon}(t)\right|_{\Omega}^{2}+\mathcal{E}\left(U_{\nu}^{\varepsilon}(t)\right)+\frac{2}{\varepsilon^{2}} \int_{\Omega} p\left(\left|U_{\nu}^{\varepsilon}(t)\right|\right) \mathrm{d} X+\frac{\alpha}{2 \gamma} \int_{0}^{t}\left|\partial_{t} U_{\nu}^{\varepsilon}(s)\right|_{\Omega}^{2} \mathrm{~d} s \\
& \leq \mathcal{E}\left(M_{0}\right)+\frac{2 \gamma \beta}{\alpha}|\boldsymbol{j}|_{\infty}^{2} \int_{0}^{t}\left|\nabla U_{\nu}^{\varepsilon}\right|_{\Omega}^{2} \mathrm{~d} s
\end{aligned}
$$

For $V \in L^{2}\left(0, T ; \mathbb{L}^{2}(\Omega)\right)$ we introduce $U=\mathcal{R}(V)$ the solution of the wave equation

$$
\left\{\begin{array}{l}
\nu^{2} \partial_{t}^{2} U-\nabla \cdot(a \nabla U)+\mathcal{B}^{\varepsilon}\left(\rho^{\nu} \star \widetilde{V}, \partial_{t} U\right) \\
=-\frac{1}{\varepsilon^{2}} \nabla_{U}(p(|U|))+\mathcal{L}(U)+\beta(\boldsymbol{j} \cdot \nabla) U \\
U(0, X)=\boldsymbol{M}_{0}(X),\left|\boldsymbol{M}_{0}(X)\right|^{2}=1 \text { a.e., } \nu^{2} \partial_{t} U(0, X)=0 .
\end{array}\right.
$$

Proceeding along the lines of the proof of our main theorem, we prove that $\mathcal{R}$ is continuous and compact from $L^{2}\left(0, T ; \mathbb{L}^{2}(\Omega)\right)$ into itself. Then as a consequence of Schauder fixed point theorem we get a solution $U_{\nu}^{\varepsilon}$ of the intermediary wave equation. We pass to the limit as $\nu \rightarrow 0$ then as $\varepsilon \rightarrow 0$ and finally prove, as in Section 3, a global existence result of weak solutions.

To end this review, let us discuss the model equations considered in [18] to describe the magnetization switching by spin-polarized current. It is given by

$$
\partial_{t} \boldsymbol{M}=-\gamma \boldsymbol{M} \times \mathcal{H}_{e}-\alpha \boldsymbol{M} \times\left(\boldsymbol{M} \times \mathcal{H}_{e}\right)-f_{\|}(t) \boldsymbol{M}
$$

where the time dependent function $f_{\|}$is related to the injected current see [18]. One observes that the saturation condition is not fulfilled by $\boldsymbol{M}$ and we have

$$
|\boldsymbol{M}(t, X)|^{2}=\left|\boldsymbol{M}_{0}(X)\right|^{2} e^{F(t)}, \quad F(t)=-\int_{0}^{t} f_{\|}(s) \mathrm{d} s .
$$


Assuming that the magnetic field $\mathcal{H}_{e}(\boldsymbol{M})$ depends linearly on $\boldsymbol{M}$ and setting $\boldsymbol{U}=$ $\boldsymbol{M} e^{-F(t)}$ we deduce that $\boldsymbol{U}$ satisfies the classical form of the LLG equation

$$
\partial_{t} \boldsymbol{U}=-\gamma(t) \boldsymbol{U} \times \mathcal{H}_{e}(\boldsymbol{U})-\alpha(t) \boldsymbol{U} \times\left(\boldsymbol{U} \times \mathcal{H}_{e}(\boldsymbol{U})\right)
$$

with the following time dependent gyromagnetic and damping coefficients

$$
\gamma(t)=\gamma e^{F(t)}, \alpha(t)=\alpha e^{2 F(t)} .
$$

One has $|\boldsymbol{U}(t)|^{2}=1$ and as usual we can transform this equation in the LLG form. We have $\boldsymbol{U} \times \partial_{t} \boldsymbol{U}=-\gamma(t) \boldsymbol{U} \times\left(\boldsymbol{U} \times \mathcal{H}_{e}(\boldsymbol{U})\right)+\alpha(t) \boldsymbol{U} \times \mathcal{H}_{e}(\boldsymbol{U})$. Consequently $-\alpha(t) \boldsymbol{U} \times\left(\boldsymbol{U} \times \mathcal{H}_{e}(\boldsymbol{U})\right)=(\alpha(t) / \gamma(t)) \boldsymbol{U} \times \partial_{t} \boldsymbol{U}-\left(\alpha^{2}(t) / \gamma(t)\right) \boldsymbol{U} \times \mathcal{H}_{e}(\boldsymbol{U})$. We get

$$
\partial_{t} \boldsymbol{U}-\frac{\alpha(t)}{\gamma(t)} \boldsymbol{U} \times \partial_{t} \boldsymbol{U}=-\frac{\alpha^{2}(t)+\gamma^{2}(t)}{\gamma(t)} \boldsymbol{U} \times \mathcal{H}_{e}(\boldsymbol{U})
$$

and $\alpha(t) / \gamma(t)=(\alpha / \gamma) e^{F(t)},\left(\alpha^{2}(t)+\gamma^{2}(t)\right) / \gamma(t)=\left(\left(\alpha^{2} e^{2 F(t)}+\gamma^{2}\right) / \gamma\right) e^{F(t)}$ and also

$$
\partial_{t} \boldsymbol{U}+\frac{\alpha(t)}{\gamma(t)} \boldsymbol{U} \times \partial_{t} \boldsymbol{U}=\frac{\alpha^{2}(t)+\gamma^{2}(t)}{\alpha(t)} \mathcal{H}_{e}(\boldsymbol{U}) .
$$

The energy estimate follows from the equality $\left|\partial_{t} \boldsymbol{U}\right|^{2}+\left(\left(\alpha^{2}(t)+\gamma^{2}(t)\right) / \alpha(t)\right) \mathcal{H}_{e}(\boldsymbol{U})$. $\partial_{t} \boldsymbol{U}=0$ which gives

$$
\mathcal{E}(\boldsymbol{U}(t))+2 \int_{0}^{t} \frac{\alpha(s)}{\alpha^{2}(s)+\gamma^{2}(s)}\left|\partial_{t} \boldsymbol{U}(s)\right|^{2} \mathrm{~d} s=\mathcal{E}\left(\boldsymbol{M}_{0}\right) .
$$

where $\mathcal{E}$ is the classical energy of the ferromagnet. Notice that we have

$$
\frac{\alpha(s)}{\alpha^{2}(s)+\gamma^{2}(s)}=\frac{\alpha}{\alpha^{2} e^{2 F(s)}+\gamma^{2}} \geq \frac{\alpha}{\alpha^{2} e^{2 M T}+\gamma^{2}}
$$

for all $s$ by assuming that $\left|f_{\|}(t)\right| \leq M$ for all $t \in[0, T]$. Hence under this hypothesis, the global existence theory of weak solutions can be performed by using the method developped here.

Acknowledgements. The authors would like to thank the referee for valuable comments and suggestions.

\section{References}

[1] Ya.B. Bazaliy, B.A. Jones and Shou-Cheng Zhang, Modification of the Landau-Lifshitz equation in the presence of a spin-polarized current in colossal- and giant-magnetoresistive materials. Phys. Rev. B, 57 (1998), R3213-3216.

[ 2 ] L. Berger, Emission of spin waves by a magnetic multilayer traversed by a current. Phys. Rev. B, 54 (1996), 9353.

[ 3 ] W.F. Brown, Micromagnetics. Interscience Publishers, New York, 1963.

[ 4 ] L.C. Evans, Weak Convergences Methods for Nonlinear Partial Differential Equations. AMS, Providence, RI, 1990. 
[ 5 ] K. Hamdache, Homogenization of layered ferromagnetic media. Preprint CMAP-495, 2002, <www.cmap.polytechnique.fr/preprint/>.

[6] K. Hamdache and D. Hamroun, Ferromagnets with biquadratic exchange coupling energy. Global existence of weak solutions. Math. Methods Appl. Sci., 28, No.12 (2005), 1403-1421.

[ 7 ] K. Hamdache and M. Tilioua, Interlayer exchange coupling for ferromagnets through spacers. SIAM J. Appl. Math., 64, No.3 (2004), 1077-1097.

[ 8 ] U. Hartman, editor, Magnetic Multilayers and Giant Magnetoresistance. Fundamentals and Industrial Applications. Springer Verlag, 2000.

[ 9 ] W.N.G. Hitchon, R.W. Chantrell and A. Rebei, Spin accumulation in ferromagnets. e-print cond-mat/0407051, 2004.

[10] J.L. Lions, Quelques Méthodes de Résolution des Problèmes aux Limites Non Linéaires. Dunod \& Gauthier-Villars, Paris, 1969.

[11] A. Shpiro, P.M. Levy and S. Zhang, Self-consistent treatment of nonequilibrium spin torques in magnetic multilayers. Phys. Rev. B, 67 (2003), 104430.

[12] J.C. Slonczewski, Current-driven excitation of magnetic multilayer. J. Magn. Magn. Mater., 159 (1996), L1.

[13] M.D. Stiles and A. Zangwill, Anatomy of spin-tranfert torque. Phys. Rev. B, 66 (2002), 014407.

[14] L. Tartar, Topics in Nonlinear Analysis. Publications Mathématiques d'Orsay 78.13, Université de Paris-Sud, Paris, 1978.

[15] G. Tatara and H. Kohno, Theory of current-driven domain wall motion: Spin transfer versus momentum transfer. Phys. Rev. Letters, 92 (2004), 086601.

[16] M. Taylor, Partial Differential Equations, III. Applied Mathematical Sciences, SpringerVerlag, New York, 1996.

[17] A. Thiaville, Y. Nakatani, J. Miltat and N. Vernier, Domain wall motion by spin-polarized current: a micromagnetic study. J. Appl. Phys., 95, No.11 (2004), 7049-7051.

[18] J.-E. Wegrowe and H.-J. Drouhin, Irreversible spin-transfer and magnetization reversal under spin-injection. e-print cond-mat/0408410, 2004.

[19] S. Zhang, P.M. Levy and A. Fert, Mechanisms of spin polarized current-driven magnetization switching. Phys. Rev. Letters, 88 (2002), 236601. 